\title{
On the over-production of turbulence beneath surface waves in Reynolds-averaged Navier-Stokes models
}

\section{Larsen, Bjarke Eltard; Fuhrman, David R.}

Published in:

Journal of Fluid Mechanics

Link to article, DOI:

10.1017/jfm.2018.577

Publication date:

2018

Document Version

Peer reviewed version

Link back to DTU Orbit

Citation (APA):

Larsen, B. E., \& Fuhrman, D. R. (2018). On the over-production of turbulence beneath surface waves in Reynolds-averaged Navier-Stokes models. Journal of Fluid Mechanics, 853, 419-460.

https://doi.org/10.1017/jfm.2018.577

\section{General rights}

Copyright and moral rights for the publications made accessible in the public portal are retained by the authors and/or other copyright owners and it is a condition of accessing publications that users recognise and abide by the legal requirements associated with these rights.

- Users may download and print one copy of any publication from the public portal for the purpose of private study or research.

- You may not further distribute the material or use it for any profit-making activity or commercial gain

- You may freely distribute the URL identifying the publication in the public portal

If you believe that this document breaches copyright please contact us providing details, and we will remove access to the work immediately and investigate your claim. 


\title{
On the over-production of turbulence beneath surface waves in Reynolds-averaged Navier-Stokes models
}

\author{
Bjarke Eltard Larsen $^{1} \dagger$, and David R. Fuhrman ${ }^{1}$ \\ ${ }^{1}$ Technical University of Denmark, Department of Mechanical Engineering, Section of Fluid \\ Mechanics, Coastal and Maritime Engineering, DK-2800 Kgs. Lyngby, Denmark
}

(Received 18-12-2017; revised 28-03-2018, 04-07-2018; accepted 09-07-2018)

In previous computational fluid dynamics studies of breaking waves, there has been a marked tendency to severely over-estimate turbulence levels, both pre- and post-breaking. This problem is most likely related to the previously described (though not sufficiently well recognized) conditional instability of widely-used turbulence models when used to close Reynolds-Averaged Navier-Stokes (RANS) equations in regions of nearly potential flow with finite strain, resulting in exponential growth of the turbulent kinetic energy and eddy viscosity. While this problem has been known for nearly 20 years, a suitable and fundamentally sound solution has yet to be developed. In this work it is demonstrated that virtually all commonly-used two-equation turbulence closure models are unconditionally, rather than conditionally, unstable in such regions. A new formulation of the $k-\omega$ closure is developed which elegantly stabilizes the model in nearly-potential flow regions, with modifications remaining passive in sheared flow regions, thus solving this longstanding problem. Computed results involving non-breaking waves demonstrate that the new stabilized closure enables nearly constant-form wave propagation over long durations, avoiding the exponential growth of the eddy viscosity and inevitable wave decay exhibited by standard closures. Additional applications on breaking waves demonstrate that the new stabilized model avoids the un-physical generation of pre-breaking turbulence which widely plagues existing closures. The new model is demonstrated to be capable of predicting accurate pre- and post-breaking surface elevations, as well as turbulence and undertow velocity profiles, especially during transition from pre-breaking to the outer surf zone. Results in the inner surf zone are similar to standard closures. Similar methods for formally stabilizing other widely-used closure models ( $k-\omega$ and $k-\varepsilon$ variants) are likewise developed, and it is recommended that these be utilized in future RANS simulations of surface waves.

\section{Introduction}

Among the most ubiquitous phenomena seen in natural aquatic environments, coastal or otherwise, are free surface waves. Such waves give rise to a highly diverse range of complexity in terms of their fluid mechanics, with the bulk region beneath non-breaking waves corresponding to nearly-potential flow, bordered by a thin (usually turbulent) boundary layer at the bottom, and often a highly complicated multi-phase (air and water) turbulent surf zone further shoreward. With the growth of computer power, computational fluid dynamics (CFD) is increasingly being used to study various freesurface wave processes, though the range of complexity mentioned above can complicate applications with a single model. Problems involving the computational study of surface

$\dagger$ Email address for correspondence: bjelt@mek.dtu.dk 
waves commonly include their simple propagation (e.g. Paulsen et al. 2014; Devolder et al. 2017), their interaction with structures (e.g. Higuera et al. 2013; Chen et al. 2014; Paulsen et al. 2014; Jacobsen et al. 2015; Schmitt \& Elsaesser 2015; Hu et al. 2016), or the highly complex of phenomena of breaking waves and surf zone dynamics.

Some of the earliest studies involving the computational study of breaking waves include Sakai et al. (1986), who used the marker-and-cell (MAC) method developed by Harlow \& Welch (1965), and Lemos (1992), who was among the first to apply the volume of fluid method (VOF), originally developed by Hirt \& Nichols (1981). Perhaps the most modelled experiments are the spilling and plunging breaking cases of Ting \& Kirby (1994, 1996). These have been widely used as validation for large eddy simulation (LES) models (e.g. Watanabe \& Saeki 1999; Hieu et al. 2004; Christensen 2006), smooth particle hydrodynamics (SPH) models (e.g. Shao 2006; Makris et al. 2016), as well as those based on Reynolds-averaged Navier-Stokes (RANS) equations coupled with various twoequation turbulence closure models, the focus of the present study. Such studies include those utilizing closures within both the $k-\varepsilon$ (e.g. Lin \& Liu 1998; Bradford 2000; Xie 2013; Hsu et al. 2015; Brown et al. 2016) and $k-\omega$ (e.g. Mayer \& Madsen 2000; Jacobsen et al. 2012, 2014; Brown et al. 2016) families.

In the RANS model studies of breaking waves mentioned above, there has been a marked and collective tendency to predict turbulence levels that are much higher than have been measured. Such over-predicted turbulence is often even most apparent prior to breaking, where (in contrast to experimental findings and intuition) computed turbulent kinetic energy levels can be the same order of magnitude as within the surf zone (see e.g. Lin \& Liu 1998; Bradford 2000; Hsu et al. 2015; Brown et al. 2016). These effects can potentially cause pre-mature wave decay, and such discrepancies can carry over well into the outer surf zone, thus affecting the computed sub-surface kinematics and subsequent surf zone dynamics as a whole. The widespread over-production of turbulence in RANS modelling of surface waves, especially prior to breaking, represents a significant and fundamental problem to their computational study. It implies that such commonlyused CFD models cannot even manage the relatively simple task of long-term wave propagation without un-physical dissipation, which should seemingly be a pre-requisite to their application on more complicated surf zone processes. Moreover, it implies that in many computational studies of the surf zone, the results have most likely been polluted even prior to the onset of the breaking process, which has usually been the very focus of study.

The underlying cause of this problem seems to not be sufficiently well recognized. For example, following long time simulations, Hsu et al. (2002) found unrealistically high turbulence in what they characterized as supposed low turbulence regions, hence being among the first to recognize this problem. They suspected that this was "due to the convection and diffusion mechanism, which transports the turbulence from the high turbulence region (e.g., the surf zone) to the low turbulence region". To combat this they included a damping mechanism on the eddy viscosity, which effectively reduced the turbulence to acceptable levels. Bradford (2011) used a $k$ - $\ell$ model and somewhat similarly found that limiting the mixing length to be less than or equal to the local flow depth maintained model stability. In a study of breaking waves using several different two-equation closures Brown et al. (2016) found that nearly all of their simulations of spilling breakers resulted in significantly over-produced turbulence prior to breaking. While they did not offer any explanation as to the underlying cause, they did recognize the detrimental effect on the local undertow profiles through comparison with results where no closure was utilized. Devolder et al. (2017) also recognized the problem of over-predicted turbulence levels beneath computed surface waves (and the related un- 
physical decay in the wave heights), and attributed this to too high eddy viscosity (hence turbulence) near the air-water interface. To combat this they included a buoyancy production term directly in the $k$-equation. While this term should likely be included in two-phase models, and it limited the excessive production of turbulence to some degree, it does not solve the fundamental problem; This can clearly be seen from their figure 7, where the computed eddy viscosity is still many orders of magnitude larger than the kinematic fluid viscosity.

Rather, the more likely explanation for the widespread over-prediction of turbulence in RANS models of surface waves was provided prior to the studies mentioned just above by Mayer \& Madsen (2000). They specifically showed through analysis that the standard $k$ - $\omega$ model of Wilcox (1988) can become unstable when applied to a region of potential flow beneath surface waves, leading to exponential growth of both the turbulent kinetic energy and eddy viscosity. Although they did not specifically analyze the $k-\varepsilon$ model, they stated that it too suffers from similar issues. Mayer \& Madsen (2000) also made an ad-hoc attempt to solve the problem by using the mean rotation, rather than the strain rate, in their production terms. This, indeed, greatly reduced the growth of the eddy viscosity and hence improved predictions of the wave breaking point relative to their unmodified model. However (as will be discussed herein), there are several fundamental deficiencies with this ad-hoc modification, and it has not been widely adopted (seemingly only by Jacobsen et al. 2012, 2014); Indeed, Mayer \& Madsen (2000) did not recommend this change as a final solution, but instead suggested further research into the problem.

Motivated largely by their suggestion, and especially by a desire to ultimately solve this long-standing problem in a more fundamentally satisfying and definitive way, the present work will re-visit the two-equation closure instability problem identified by Mayer \& Madsen (2000), who strictly proved conditional instability of the $k$ - $\omega$ closure model. Their analysis will be briefly reviewed and extended to prove that this model is, in fact, unconditionally unstable for the conditions they considered, with a predictable asymptotic exponential growth rate when applied to a region of nearly-potential flow having finite strain. Building directly on the analysis, we will likewise demonstrate how such models can be simply and elegantly stabilized, in a manner that will importantly remain passive in other sheared regions of interest. The significant advantages to utilizing the new stabilized closure model will be demonstrated directly through examples involving simulations of non-breaking and breaking waves.

The present paper is organized as follows: Stability analysis of several RANS turbulence closure models will be performed in Section 2, including a review of the work of Mayer \& Madsen (2000). The analysis in the main text will focus on $k-\omega$ closure models, including the standard model of Wilcox (1988), the ad-hoc modification of Mayer \& Madsen (2000), as well as the more recent version presented by Wilcox (2006). Building further from the analysis, this will subsequently lead to development of a new and formally stable $k$ - $\omega$ closure model that is otherwise compatible with these earlier versions. The significant advantages of utilizing the stabilized model will be demonstrated in Section 3, for problems involving simple surface wave propagation, as well as the simulation of the spilling breaking wave experiment of Ting \& Kirby (1994), where the problem of overproduction of turbulence leading up to breaking is known to be especially pronounced.

Similar analysis of several other widely-utilized two-equation turbulence closure models ( $k-\omega \mathrm{SST}$, standard $k-\varepsilon$, and RNG $k-\varepsilon$ models) is provided in Appendix A for completeness. The analysis therein demonstrates that these models are likewise unconditionally unstable in the same sense as are those considered in the main text. Furthermore, we demonstrate that they too can be formally stabilized with similar, relatively simple, modifications. 


\section{Stability analysis of RANS turbulence closure models beneath nearly-potential flow waves}

\subsection{Turbulence closure model}

For many problems in fluid mechanics, it is neither practical nor computationally feasible, to resolve all necessary scales for direct numerical simulation (DNS) or even large eddy simulation (LES), both of which inevitably require high resolution of three spatial dimensions. As an alternative, it is often necessary to instead work within the confines of Reynolds-averaged Navier-Stokes (RANS) equations, thereby necessitating a separate closure model for describing the effects of turbulence on the mean flow. As a suitable description of turbulence for the present purposes, we will adopt a generalized version of the widely-used $k$ - $\omega$ model, comprised of the following transport equations for the turbulent kinetic energy density $k=1 / 2\left(\overline{u_{i}^{\prime} u_{i}^{\prime}}\right)$ :

$$
\frac{\partial \rho k}{\partial t}+u_{j} \frac{\partial \rho k}{\partial x_{j}}=\rho P_{k}-\rho P_{b}-\rho \beta^{*} k \omega+\frac{\partial}{\partial x_{j}}\left[\left(\mu+\rho \sigma^{*} \frac{k}{\omega}\right) \frac{\partial k}{\partial x_{j}}\right]
$$

and the specific dissipation rate $\omega$ :

$$
\frac{\partial \rho \omega}{\partial t}+u_{j} \frac{\partial \rho \omega}{\partial x_{j}}=\rho P_{\omega}-\rho \beta \omega^{2}+\rho \frac{\sigma_{d}}{\omega} \frac{\partial k}{\partial x_{j}} \frac{\partial \omega}{\partial x_{j}}+\frac{\partial}{\partial x_{j}}\left[\left(\mu+\rho \sigma \frac{k}{\omega}\right) \frac{\partial \omega}{\partial x_{j}}\right] .
$$

Here $u_{i}$ are the mean components of the velocity, $x_{j}$ are the Cartesian coordinates, $\mu=\rho \nu$ is the dynamic molecular viscosity, $\nu$ is the kinematic viscosity, $\rho$ is the density, $t$ is time, and

$$
\tau_{i j}=-\overline{u_{i}^{\prime} u_{j}^{\prime}}=2 \nu_{T} S_{i j}-\frac{2}{3} k \delta_{i j}
$$

is the Reynolds stress tensor, expressed in accordance with the Boussinesq approximation. Here the overbar signifies time (ensemble) averaging, a prime superscript denotes turbulent (fluctuating) components, $\delta_{i j}$ is the Kronecker delta,

$$
S_{i j}=\frac{1}{2}\left(\frac{\partial u_{i}}{\partial x_{j}}+\frac{\partial u_{j}}{\partial x_{i}}\right)
$$

is the mean strain rate tensor, and

$$
\nu_{T}=\frac{k}{\tilde{\omega}}
$$

is the eddy viscosity. The shear production term for $k$ is

$$
P_{k}=\tau_{i j} \frac{\partial u_{i}}{\partial x_{j}}=p_{0} \nu_{T}, \quad p_{0}=2 S_{i j} S_{i j} .
$$

Similarly, the buoyancy production for $k$ is formulated as

$$
P_{b}=-\frac{g_{i}}{\rho} \overline{\rho^{\prime} u_{i}^{\prime}}=p_{b} \nu_{T}, \quad p_{b}=\alpha_{b}^{*} N^{2}, \quad N^{2}=\frac{g_{i}}{\rho} \frac{\partial \rho}{\partial x_{i}},
$$

where $\left(g_{1}, g_{2}, g_{3}\right)=(0,0,-g)$ is gravitational acceleration and $N$ is the the Brunt-Vaisala frequency. The production of $\omega$ is likewise taken as

$$
P_{\omega}=\alpha \frac{\omega}{k} \frac{\tilde{\omega}}{\tilde{\tilde{\omega}}} P_{k}=\alpha \frac{\omega}{\tilde{\tilde{\omega}}} p_{0} .
$$

It is emphasized that the basic form of the shear turbulence production term (i.e. $P_{k}=$ $\left.\tau_{i j} \partial u_{i} / \partial x_{j}\right)$ should be considered sacred, as it can be theoretically derived through the Reynolds-averaging process; As the Reynolds stress $\tau_{i j}$ appears both here, as well as 
in the governing RANS equations, it is imperative that they be kept identical. The production of the specific dissipation rate $P_{\omega}$, on the other hand, is essentially empirical in nature (based on dimensional analysis, hence necessitating the closure coefficient $\alpha$ on this term). It is hence not theoretically tied to the RANS equations, and may therefore be treated with considerably more freedom.

The equations above can be considered a generalized form of the Wilcox (2006) turbulence closure model. In addition to his basic model, we have specifically added the previously defined buoyancy production term $P_{b}$ in (2.1) for potential application to two-phase (air-water) flows. A similar term was implemented for the $k-\varepsilon$ model by e.g. Rodi (1987), Burchard (2002) and Ruessink et al. (2009), for the $k$ - $\omega$ model by e.g. Umlauf et al. (2003) and Fuhrman et al. (2013) as well as for the $k$ - $\omega$ SST model by Devolder et al. (2017). We have likewise incorporated additional freedom via the introduction of two (rather than the usual one) utility variables $\tilde{\omega}$ and $\tilde{\tilde{\omega}}$ within (2.5) and (2.8). These represent potential stress limited versions of $\omega$, to be determined and taken advantage of in what follows. The generalized form presented above is convenient, as it can be reduced to a number of common variations of the $k-\omega$ model in the literature, with suitable selections of $\tilde{\omega}, \tilde{\tilde{\omega}}$, and closure coefficients. Throughout the present work (unless noted otherwise) we will adopt the closure coefficients of Wilcox $(2006,2008)$ : $\alpha=0.52, \beta=0.0708$ (constant for two-dimensional flows), $\beta^{*}=0.09, \sigma=0.5, \sigma^{*}=0.6$, $\sigma_{d o}=0.125$, with

$$
\sigma_{d}=H\left(\frac{\partial k}{\partial x_{j}} \frac{\partial \omega}{\partial x_{j}}\right) \sigma_{d o},
$$

where $H(\cdot)$ is the Heaviside step function, which takes a value of unity if the argument is positive and zero otherwise. Additionally, we adopt the value $\alpha_{b}^{*}=1.36$, which is derived in Appendix B.

\subsection{Turbulence production beneath potential flow waves}

Let us now briefly review the production of turbulence beneath potential flow progressive waves, as originally described by Mayer \& Madsen (2000). Following their work, and for the sake of simplicity, let us consider the velocity fields given by Stokes first-order wave theory, where $z=0$ is measured from the bed:

$$
\begin{aligned}
& u=\frac{H \sigma}{2} \frac{\cosh \left(k_{w} z\right)}{\sinh \left(k_{w} h\right)} \cos \left(k_{w} x-\sigma t\right), \\
& w=\frac{H \sigma}{2} \frac{\sinh \left(k_{w} z\right)}{\sinh \left(k_{w} h\right)} \sin \left(k_{w} x-\sigma t\right) .
\end{aligned}
$$

Here $\sigma$ is the angular frequency, $k_{w}$ is the wave number, $h$ is the water depth and $H$ is the wave height. If these velocity fields are inserted into (2.6), after period-averaging, there will be turbulent kinetic energy production corresponding to:

$$
\left\langle p_{0}\right\rangle=\frac{k_{w}^{2} H^{2} \sigma^{2}}{2} \frac{\cosh \left(2 k_{w} z\right)}{\sinh ^{2}\left(k_{w} h\right)} .
$$

Note that after further depth-averaging, this becomes

$$
\left\langle\left\langle p_{0}\right\rangle\right\rangle=\frac{k_{w}^{2} H^{2} \sigma^{2}}{2 k_{w} h \tanh \left(k_{w} h\right)} .
$$

Hence, the above demonstrates that there will be a non-zero production of turbulent kinetic energy in a potential flow region beneath surface waves, provided that the eddy viscosity is finite. 


\subsection{Analysis of the standard Wilcox (1988) $k-\omega$ model}

Having established that standard methods for achieving turbulence closure will potentially result in finite turbulence production in a region of potential flow, let us now conduct a formal stability analysis of several widely-used closure models. For this purpose, consider a region of nearly-potential flow, such that

$$
p_{\Omega}=2 \Omega_{i j} \Omega_{i j} \ll p_{0}, \quad \Omega_{i j}=\frac{1}{2}\left(\frac{\partial u_{i}}{\partial x_{j}}-\frac{\partial u_{j}}{\partial x_{i}}\right)
$$

in a fluid of constant density (hence $P_{b}=p_{b}=0$ ), where $\Omega_{i j}$ is the mean rotation rate tensor and $p_{0}$ is assumed fixed at some finite value. Following Mayer \& Madsen (2000), diffusive and convective terms will be neglected in the analysis for the sake of simplicity, which is justifiable in the region above the bottom boundary layer. In this case the governing generalized turbulence model equations (2.1) and (2.2) reduce to

$$
\begin{aligned}
& \frac{\partial k}{\partial t}=\frac{k}{\tilde{\omega}} p_{0}-\beta^{*} \omega k, \\
& \frac{\partial \omega}{\partial t}=\alpha \frac{\omega}{\tilde{\tilde{\omega}}} p_{0}-\beta \omega^{2} .
\end{aligned}
$$

Let us begin by analyzing what will be deemed the standard (Wilcox 1988) $k$ - $\omega$ model. This corresponds to setting $\omega=\tilde{\omega}=\tilde{\tilde{\omega}}$ in the above leading to the further reduced equations

$$
\begin{gathered}
\frac{\partial k}{\partial t}=\frac{k}{\omega} p_{0}-\beta^{*} \omega k \\
\frac{\partial \omega}{\partial t}=\alpha p_{0}-\beta \omega^{2} .
\end{gathered}
$$

This corresponds to the same form as considered by Mayer \& Madsen (2000). (Note that the closure coefficients in the Wilcox (1988) model are slightly different from those used here, but have no qualitative influence on the analysis.) Mayer \& Madsen (2000) proved conditional instability of this model, stating that if at any instant

$$
\omega \leqslant \sqrt{\frac{1-\alpha}{\beta^{*}-\beta} p_{0}}=5 \sqrt{p_{0}}
$$

then the turbulence model will become unstable, resulting in exponential growth of the eddy viscosity.

In what follows, we will extend their analysis, and formally prove that the simplified model, subject to the conditions described above, is in fact unconditionally unstable. Moreover, we will establish a methodology to analytically determine the asymptotic unstable growth rate. From inspection of (2.18), it is seen that this equation is de-coupled from the $k$ equation (2.17). Hence, regardless of its initial value, $\omega$ will ultimately evolve to a constant such that $\partial \omega / \partial t=0$, corresponding in this case to

$$
\omega_{\infty}=\sqrt{\frac{p_{0} \alpha}{\beta}} \approx 2.71 \sqrt{p_{0}} .
$$

As this satisfies the constraint (2.19), this slight extension thus proves that the model is, indeed, unconditionally unstable; This stronger finding has apparently also been arrived at independently by Mayer ca. 2001 (personal communication, October 31, 2017). Moreover, once the specific dissipation rate reaches its asymptotic value $\omega=\omega_{\infty}$, the $k$ 
equation effectively becomes linearised, ultimately leading to equations of the form

$$
\frac{1}{k} \frac{\partial k}{\partial t}=\frac{1}{\nu_{T}} \frac{\partial \nu_{T}}{\partial t}=\Gamma_{\infty}
$$

having solutions such that $k \sim \nu_{T} \sim \exp \left(\Gamma_{\infty} t\right)$, where $\Gamma_{\infty}$ is the asymptotic unstable growth rate. For the standard $k-\omega$ model under consideration this works out to be

$$
\Gamma_{\infty}=\left(\beta-\alpha \beta^{*}\right) \sqrt{\frac{p_{0}}{\alpha \beta}} \approx 0.125 \sqrt{p_{0}} .
$$

As an independent check of the analysis above, we have performed several numerical simulations of (2.17) and (2.18). As a demonstration we will consider a case with initial conditions $\omega=\omega_{0}=100 \sqrt{p_{0}}$, such that the turbulence scales are initially well separated from those of the mean flow, and $k=k_{0}=10 \nu \sqrt{p_{0}}$ such that initially $\nu_{T} / \nu=0.1$. Figure 1 shows the simulated temporal development of $k / k_{0}, \omega / \sqrt{p_{0}}$ and $\nu_{T} / \nu$. Included in the figure as the dashed lines are the predicted asymptotic exponential growth rates for $k$ and $\nu_{T}$ from the above analysis, as well as the predicted asymptotic value $\omega_{\infty}$. As can be seen $\omega$ quickly tends towards $\omega_{\infty}$, and once this occurs $k$ and $\nu_{T}$ (which were initially declining) start growing exponentially at precisely the growth rate $\Gamma_{\infty}$ predicted above.

\subsection{Analysis of the modified Mayer \& Madsen (2000) $k$ - $\omega$ model}

In an effort to combat the un-physical growth of turbulence in their CFD simulation of breaking waves, Mayer \& Madsen (2000) made an ad-hoc modification of their production terms $\left(P_{k}\right.$ and $P_{\omega}$, though they did not modify the eddy viscosity outside these terms) such that they were based on the vorticity, rather than the strain rate. They did not formally analyze the resulting turbulence closure model for stability, and this will therefore be investigated here. In the context of our simplified analysis, this ad-hoc modification is equivalent to setting

$$
\tilde{\omega}=\tilde{\tilde{\omega}}=\frac{p_{0}}{p_{\Omega}} \omega
$$

whence (2.15) and (2.16) become

$$
\begin{gathered}
\frac{\partial k}{\partial t}=\frac{k}{\omega} p_{\Omega}-\beta^{*} \omega k, \\
\frac{\partial \omega}{\partial t}=\alpha p_{\Omega}-\beta \omega^{2} .
\end{gathered}
$$

These are identical to $(2.17)$ and $(2.18)$, but with $p_{0}$ now replaced by $p_{\Omega}$. Hence, without requiring further analysis, it is evident that in this model $\omega$ will tend asymptotically to

$$
\omega_{\infty}=\sqrt{\frac{p_{\Omega} \alpha}{\beta}} \approx 2.71 \sqrt{p_{\Omega}}
$$

at which point the unstable growth rate for $k$ and $\nu_{T}$ will be

$$
\Gamma_{\infty}=\left(\beta-\alpha \beta^{*}\right) \sqrt{\frac{p_{\Omega}}{\alpha \beta}} \approx 0.125 \sqrt{p_{\Omega}} .
$$

Thus, even with this alteration, since the vorticity would never be exactly zero in a CFD model involving surface waves (due to both numerical error and/or imposed boundary conditions) the resulting model is still formally unconditionally unstable, though the asymptotic growth rate would be significantly reduced compared to the standard $k-\omega$ model, since it is assumed that $p_{\Omega} \ll p_{0}$. 

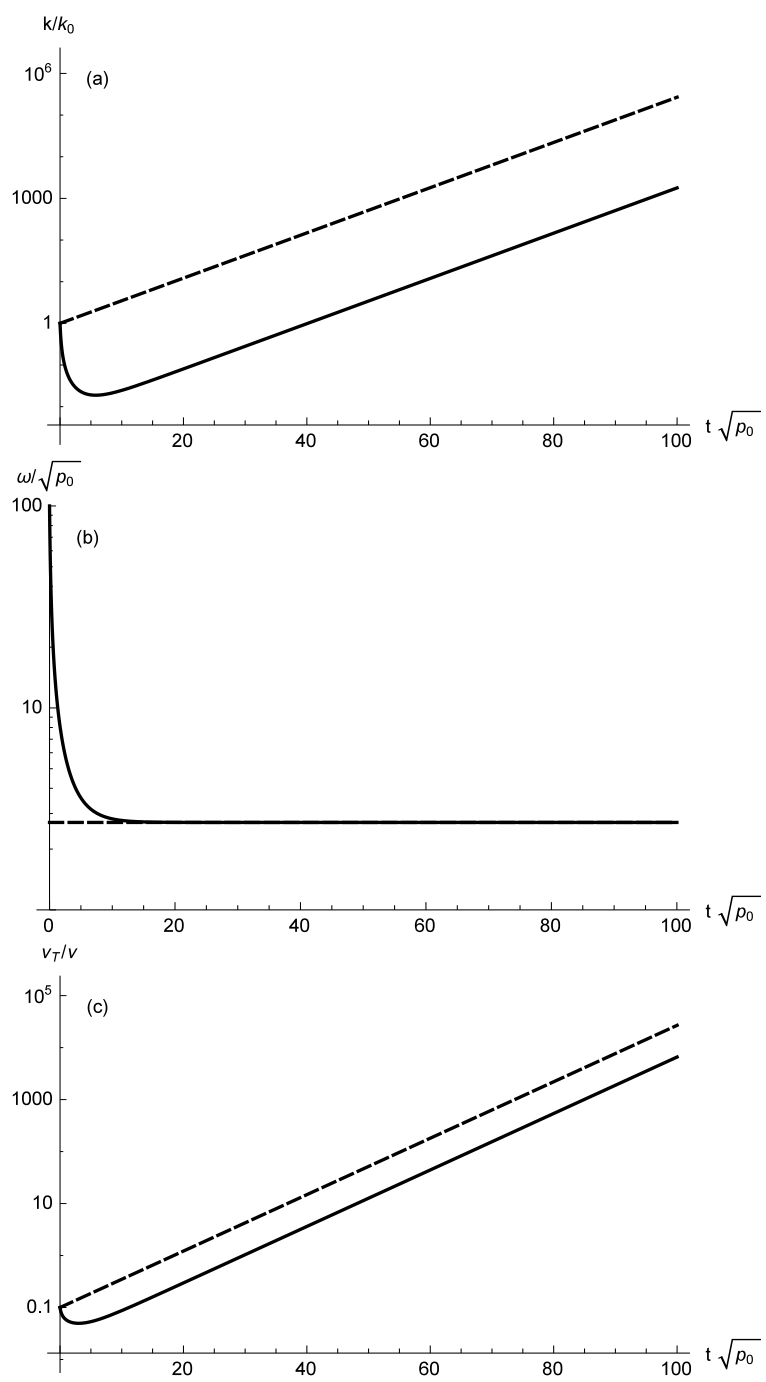

Figure 1. Simulated development (full lines) of (a) $k / k_{0}$, (b) $\omega / \sqrt{p_{0}}$ and (c) $\nu_{T} / \nu$ for the Wilcox (1988) closure model. Dashed lines indicate predicted asymptotic exponential growth rates in (a) $k$ and (c) $\nu_{T}$, as well as (b) the predicted asymptotic value $\omega=\omega_{\infty}$.

Independent confirmation of the analysis above is provided via numerical solution of the reduced governing equations, maintaining the same initial conditions as before and now with $p_{\Omega} / p_{0}=0.01$. The resulting evolution of the eddy viscosity is depicted in figure 2(a). Again this first declines, before ultimately growing exponentially at precisely the asymptotic rate predicted above.

The ad-hoc modification used by Mayer \& Madsen (2000) represents an interesting first attempt to control the instability that they identified, and which was expanded upon in the preceding sub-section. Nevertheless, it cannot be considered a fundamentally viable solution for several reasons. First, though weaker, the model is still formally unconditionally unstable, as proved above. Second, Mayer \& Madsen (2000) effectively utilized different $\tilde{\omega}$ in the production terms and in the eddy viscosity outside of these terms, which is in direct violation of the Boussinesq approximation (2.3); This is equivalent 

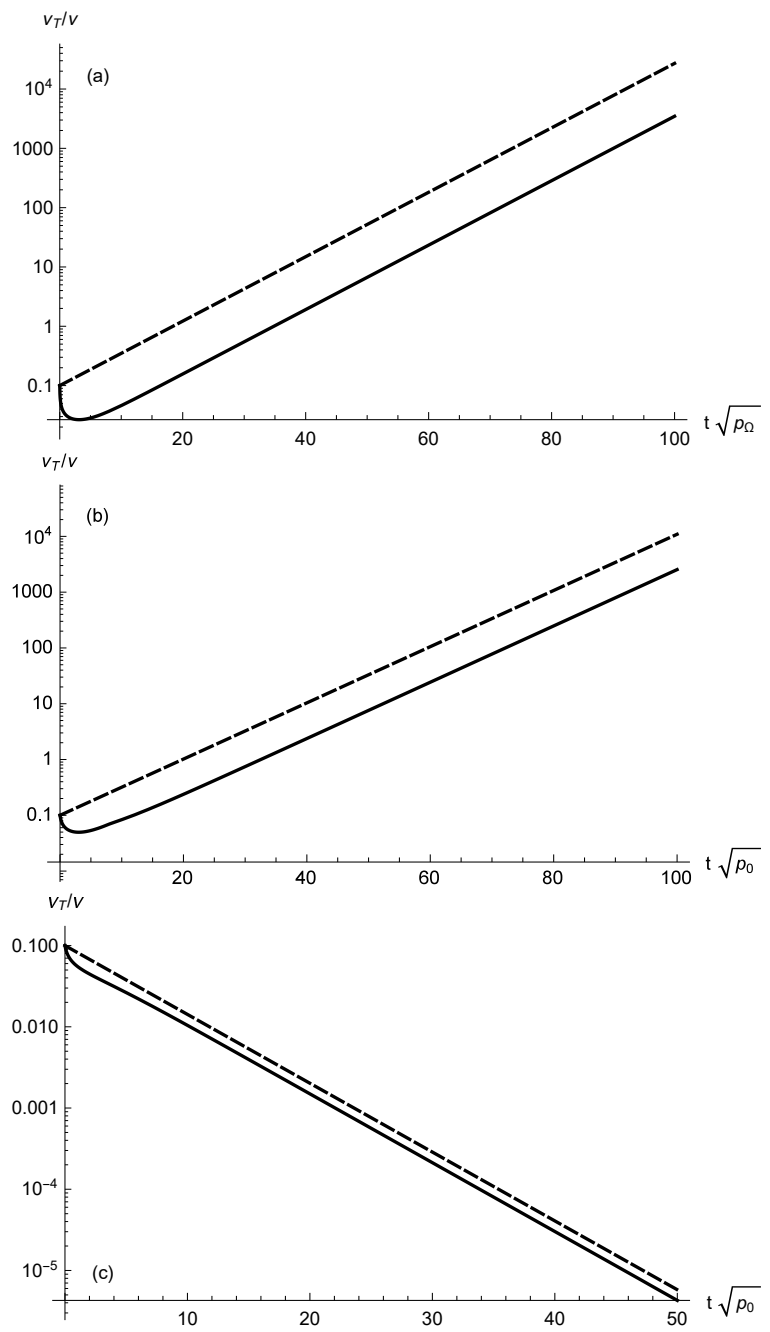

Figure 2. Simulated development of $\nu_{T} / \nu$ (full lines) compared to the predicted asymptotic exponential growth (dashed lines) for the (a) Mayer \& Madsen (2000) $k$ - $\omega$ closure, (b) Wilcox (2006) $k-\omega$ closure, and (c) present $k-\omega$ closure with $\lambda_{1}=0$ and $\lambda_{2}=0.05$.

to simultaneously utilizing two different definitions of the Reynolds stress tensor $\tau_{i j}$. Third, it is again emphasized that the turbulence production term is theoretical in nature (derived directly from Reynolds averaging), and hence rightly ought to be based on the strain rate rather than the vorticity, at least if the standard Boussinesq approximation is utilized. While in simple uniform boundary layer flows these may be equal, in more complex flows they can be quite different. (For example, in the forthcoming simulation of spilling breaking waves we have found that they can differ by a factor of 1-10 in most of the surf zone.) Hence, in the context of surface waves this modification must be considered intrusive, resulting in a significantly altered turbulence production term that is applied globally i.e. even in sheared flow regions of primary interest (e.g. the surf zone), where the original model should be maintained. This is evidenced directly by the work of Jacobsen, Fuhrman \& Fredsøe (2012), who utilized the modified production terms of Mayer \& Madsen (2000) to simulate spilling breaking waves, and found that it was necessary to alter one of the fundamental closure coefficients in isolation (from 
$\alpha=0.52$ to 0.4$)$ to obtain reasonable undertow profiles. This is likewise problematic, since as shown e.g. in Wilcox (2006), the closure coefficients are carefully tuned, and are, among other things, related to the von Karman constant $\kappa$ according to

$$
\alpha=\frac{\beta}{\beta^{*}}-\sigma \frac{\kappa^{2}}{\sqrt{\beta^{*}}} .
$$

Hence, the alteration just mentioned corresponds to a model yielding $\kappa \approx 0.482$ rather than the accepted $\kappa \approx 0.4$, and will therefore be inaccurate for simple steady uniform boundary layer flows. Indeed, it must be pointed out that (likely in recognition of some of the concerns presented above) Mayer \& Madsen (2000) rightly concluded that "at this stage we do not recommend this modification as generally applicable and instead some new fundamental analysis and development seems necessary," largely inspiring the present work.

\subsection{Analysis of the Wilcox (2006) $k$ - $\omega$ model}

As a third alternative, let us now similarly consider the stability of the Wilcox (2006) $k-\omega$ closure model. In this closure model Wilcox (2006) added, among other things, a stress limiting feature, such that

$$
\tilde{\omega}=\tilde{\tilde{\omega}}=\max \left[\omega, \lambda_{1} \sqrt{\frac{p_{0}}{\beta^{*}}}\right]
$$

where he suggested $\lambda_{1}=7 / 8=0.875$. This feature essentially limits the resulting eddy viscosity in regions where turbulence production exceeds the dissipation, and has been shown to result in larger separation bubbles and greatly improve incompressible and transonic-flow predictions relative to models without this feature.

If the first argument in the limiter (2.29) dominates, then $\tilde{\omega}=\tilde{\tilde{\omega}}=\omega$ and the model becomes identical to that analyzed in $\S 2.3$, which was already proven to be unconditionally unstable in a nearly-potential flow with finite $p_{0}$. Alternatively, if the second (stress limiting) argument dominates, then (2.15) and (2.16) become

$$
\begin{aligned}
& \frac{\partial k}{\partial t}=\frac{k}{\lambda_{1}} \sqrt{\beta^{*} p_{0}}-\beta^{*} \omega k, \\
& \frac{\partial \omega}{\partial t}=\frac{\alpha \omega}{\lambda_{1}} \sqrt{\beta^{*} p_{0}}-\beta \omega^{2} .
\end{aligned}
$$

As before, setting the right-hand-side of (2.31) equal to zero, we now find that $\omega$ will tend to the asymptotic value

$$
\omega_{\infty}=\frac{\alpha}{\lambda_{1} \beta} \sqrt{\beta^{*} p_{0}} \approx 2.52 \sqrt{p_{0}} .
$$

Inserting this value for $\omega$ back into (2.30) then leads directly to linearised expressions of the form (2.21), where the unstable growth rate is

$$
\Gamma_{\infty}=\frac{\left(\beta-\alpha \beta^{*}\right)}{\lambda_{1} \beta} \sqrt{\beta^{*} p_{0}} \approx 0.116 \sqrt{p_{0}}
$$

Hence, this model is likewise formally unconditionally unstable in the situation considered, though the stress limiter notably reduces the unstable growth rate slightly relative to the standard $k-\omega$ model.

Independent confirmation of the analysis above is provided via numerical solution of (2.15) and (2.16) while invoking (2.29), maintaining the same initial conditions for $\omega$ and $\nu_{T}$ as before. The resulting evolution of the eddy viscosity is depicted in figure 2(b). As 
before, following the initial decline, the eddy viscosity grows exponentially at the rate predicted above.

\subsection{Analysis of a new stabilized closure}

In this section an elegant and simple solution to the instability considered above, which seems to widely plague most existing two-equation closure options (all that the authors have analyzed), will be presented. We seek a solution to this long-standing problem that will be otherwise un-intrusive i.e. such that the resulting model will default to an existing closure in sheared flow regions, while still formally curing the instability in regions of nearly-potential flow. At the same time the solution should remain true to the theoretically based terms in the $k$ equation, maintain full consistency with the Boussinesq approximation, and not require alteration of any fundamental closure coefficients. We further aim for the solution to be readily adaptable to similarly stabilize other twoequation closures in wide use.

As just shown in $\S 2.5$, the stress limiting feature in the Wilcox (2006) model reduces the unstable growth rate, relative to the standard $k-\omega$ model. Working within this established feature is therefore a natural place to attempt to remedy this problem. To both formally stabilize the model and generally improve the CFD simulation of surface waves with RANS models, we propose the following modifications to the stress limiting features. First, we propose to generalize $\tilde{\tilde{\omega}}$ slightly such that:

$$
\tilde{\tilde{\omega}}=\max \left[\omega, \lambda_{1} \sqrt{\frac{p_{0}-p_{b}}{\beta^{*}}}\right],
$$

where we have included buoyancy production for potential two-phase (air and water) flow applications, for the sake of full consistency with (2.1). Obviously, this will not affect the formal stability of the model, as constant density (hence $p_{b}=0$ ) is assumed in the analysis. Second (and much more importantly in the present context), to formally stabilize the instability considered at length above, we propose the following modification to $\tilde{\omega}$ :

$$
\tilde{\omega}=\max \left[\tilde{\tilde{\omega}}, \lambda_{2} \frac{\beta}{\beta^{*} \alpha} \frac{p_{0}}{p_{\Omega}} \omega\right]
$$

where $\lambda_{2} \ll 1$ is an additional stress limiter coefficient, the physical meaning of which will be made explicitly clear in what follows. Note that the new addition to the limiter in (2.35) is, by design, un-intrusive, as it will become active only in a region of nearlypotential flow where $p_{0} \gg p_{\Omega}$. Moreover, note that (for single-phase incompressible flows) if $\lambda_{2}=0$ the model becomes equivalent to the Wilcox (2006) model, whereas if $\lambda_{1}=\lambda_{2}=0$ this model becomes equivalent to the standard $k-\omega$ model of Wilcox (1988). It is hence fully compatible with the other standard closures that have been specifically considered. (Note that to avoid any possibility of dividing by zero, the denominator in (2.35) can be implemented numerically as $p_{\Omega}+\xi$, where $\xi$ is a small number near machine precision, though we strictly analyze the form above for the sake of elegance.)

To demonstrate the formal asymptotic stability of the proposed new model, let us first repeat the above analysis of the standard Wilcox (1988) model (momentarily setting $\lambda_{1}=0$, hence $\tilde{\tilde{\omega}}=\omega$ ), but now with the new limiter active in $\tilde{\omega}$. In this case, the $k$ equation (2.15) becomes

$$
\frac{\partial k}{\partial t}=\frac{\alpha \beta^{*}}{\lambda_{2} \beta} \frac{k p_{\Omega}}{\omega}-\beta^{*} \omega k
$$

whereas the $\omega$ equation from (2.16) remains equivalent to (2.18), and hence $\omega$ will ultimately evolve to $\omega_{\infty}$ from (2.20). Inserting this value into (2.36) leads to linearized 
expressions of the form (2.21), where the exponential growth rate is

$$
\Gamma_{\infty}=\sqrt{\frac{p_{0} \alpha}{\beta}} \beta^{*}\left(\frac{p_{\Omega}}{\lambda_{2} p_{0}}-1\right) .
$$

From this it can be seen that the new turbulence closure model will be formally stable (i.e. $\left.\Gamma_{\infty} \leqslant 0\right)$ provided that

$$
\frac{p_{\Omega}}{p_{0}} \leqslant \lambda_{2}
$$

This hence provides a clear physical meaning for the added stress limiter coefficient $\lambda_{2}$, as it defines the threshold of $p_{\Omega} / p_{0}$ identifying a region as effectively potential flow. Note also that at the pure potential flow limit where $p_{\Omega}=0$, the growth rate is $\Gamma_{\infty}=-\beta^{*} \sqrt{p_{0} \alpha / \beta}=-\beta^{*} \omega_{\infty} \approx-0.244 \sqrt{p_{0}}$, and any turbulent kinetic energy will decay similar to how it would in a quiescent fluid.

If instead, we repeat the analysis of the Wilcox (2006) model (now retaining $\lambda_{1}$ ), then the $k$ equation will remain equivalent to $(2.36)$, whereas the $\omega$ equation will be equivalent to $(2.31)$, and hence $\omega$ will tend towards $\omega_{\infty}$ in (2.32). Inserting this value as a constant within (2.36) then similarly leads to linearized expressions of the form (2.21), where the unstable growth rate is

$$
\Gamma_{\infty}=\sqrt{p_{0}} \beta^{*}\left(\frac{p_{\Omega} \lambda_{1}}{p_{0} \lambda_{2} \sqrt{\beta^{*}}}-\frac{\alpha \sqrt{\beta^{*}}}{\lambda_{1} \beta}\right) .
$$

This will thus be stable provided that

$$
\frac{p_{\Omega}}{p_{0}} \leqslant \lambda_{2} \frac{\alpha \beta^{*}}{\lambda_{1}^{2} \beta} \approx 0.863 \lambda_{2},
$$

where the standard coefficient values have been inserted. As the lead coefficient is near unity, it is seen that this further generalization does not greatly affect the previously mentioned physical interpretation of $\lambda_{2}$. Note also that once $\omega=\omega_{\infty}$ if $\lambda_{1}<\sqrt{\alpha \beta^{*} / \beta} \approx$ 0.813 then the first argument will inevitably dominate in (2.34), and the threshold for stability will again be given by (2.38).

It should be noted that in uniform boundary layer flows $p_{0}=p_{\Omega}$, and thus the new limiter will be completely inactive. Moreover, in other more complicated sheared flow regions (e.g. both in the surf zone and the surface boundary layer region near the airwater interface) $p_{\Omega}$ and $p_{0}$ will typically be the same order of magnitude, and the new limiter will similarly remain inactive. Hence, the new closure will, by design, effectively reduce to a standard closure, except in a region of nearly-potential flow (clearly defined by the choice of $\lambda_{2}$ ), where such existing standard methods are inherently unconditionally unstable. The value of $\lambda_{2}$ should naturally be small, but also large enough to work for practical applications since $p_{\Omega}$ can always be expected to have a small, but finite, value in the discretized world. Throughout this work $\lambda_{2}=0.05$ is used to achieve such a balance.

The stabilization of the new model presented above has been confirmed via numerical simulation of (2.15) and (2.16) while invoking (2.34) and (2.35), maintaining the same initial conditions as before. An example (with $\lambda_{1}=0$ and $\lambda_{2}=0.05$ ) depicting the temporal evolution of the eddy viscosity is provided in figure $2(\mathrm{c})$. Consistent with the analysis above, exponential decay, rather than growth, is now observed. Thus, the new closure model should remain stable in a region of nearly-potential flow with finite strain, in contrast to all of the other models considered previously. 


\begin{tabular}{cccc}
\hline Model & Reference & $\approx \omega_{\infty}$ & $\approx \Gamma_{\infty}$ \\
\hline Standard $k-\omega$ & Wilcox (1988) & $2.71 \sqrt{p_{0}}$ & $0.125 \sqrt{p_{0}}$ \\
$k-\omega$ & Mayer \& Madsen (2000) & $2.71 \sqrt{p_{\Omega}}$ & $0.125 \sqrt{p_{\Omega}}$ \\
$k-\omega$ & Wilcox (2006) & $2.52 \sqrt{p_{0}}$ & $0.116 \sqrt{p_{0}}$ \\
$k-\omega$ SST & Menter (1994) & $2.72 \sqrt{p_{0}}$ & $0.066 \sqrt{p_{0}}$ \\
Standard $k-\varepsilon$ & Launder \& Sharma (1974) & $2.31 \sqrt{p_{0}}$ & $0.226 \sqrt{p_{0}}$ \\
RNG $k-\varepsilon$ & Yakhot et al. (1991) & $2.70 \sqrt{p_{0}}$ & $0.142 \sqrt{p_{0}}$ \\
\hline
\end{tabular}

TABLE 1. Summary of asymptotic $\omega$ values $\left(\omega_{\infty}\right)$ and unstable exponential growth rates $\left(\Gamma_{\infty}\right)$ for analyzed existing two-equation turbulence closures.

\subsection{Analysis of other existing closure models}

The formal asymptotic stability of several other widely-utilized two-equation turbulence closure models is similarly considered in Appendix A. These include: the $k-\omega$ SST model originally formulated by Menter (1994), the standard $k-\varepsilon$ model of Launder \& Sharma (1974), and the RNG $k-\varepsilon$ model developed by Yakhot et al. (1991). It turns out that all of these basic two-equation models are similarly unconditionally unstable for the same conditions as analyzed above. This is demonstrated analytically, as well as via independent numerical simulations of the reduced governing equations. The resulting asymptotic values for $\omega_{\infty}$ and the unstable growth rates $\Gamma_{\infty}$ for each of the pre-existing closure models analyzed in the present work are summarized in table 1.

Fortunately, and in line with the goals set forth above, these other widely-utilized closure models can also be formally stabilized via simple modifications to their stress limiting features, in a similar manner as presented in the preceding sub-section. The necessary modifications to stabilize each model are described in full detail in Appendix A. With these modifications, asymptotic exponential decay (rather than growth) in the turbulent kinetic energy and eddy viscosity is proved analytically, as well as independently demonstrated through numerical simulation of the reduced modified model equations, similar to the above.

To avoid the potential for excessive and un-physical over-production of turbulence in nearly-potential flow regions, it is recommended that these (or otherwise formally stabilized and un-intrusive) modified closure models be utilized in any future CFD simulations of surface waves with two-equation RANS closure models, the significant and fundamental benefits of which will be demonstrated in the next section.

\section{Numerical simulation of surface waves}

The advantages of utilizing a formally stable closure model will now be demonstrated directly through the CFD simulation of surface water waves. Essential details of the computational model are provided in the next sub-section, which will be followed by test cases involving the simulation of both non-breaking and breaking waves.

\subsection{Model description}

For the purposes of CFD simulation, surface water waves will be considered in the context of two-phase (air and water) flow. For this purpose the turbulence model defined 
by (2.1) and (2.2) will be used to close a CFD model solving incompressible Reynoldsaveraged Navier-Stokes (RANS) equations

$$
\frac{\partial \rho u_{i}}{\partial t}+u_{j} \frac{\partial \rho u_{i}}{\partial x_{j}}=-\frac{\partial p^{*}}{\partial x_{i}}-g_{j} x_{j} \frac{\partial \rho}{\partial x_{i}}+\frac{\partial}{\partial x_{j}}\left(2 \mu S_{j i}+\tau_{i j}\right),
$$

and the local continuity equation

$$
\frac{\partial u_{i}}{\partial x_{i}}=0
$$

In the above $p^{*}$ is the pressure in excess of hydrostatic. A scalar field $\gamma$ is used to track the two fluids, where $\gamma=0$ represents pure air and $\gamma=1$ pure water, with any intermediate value representing a mixture. The distribution of $\gamma$ is governed by an advection equation

$$
\frac{\partial \gamma}{\partial t}+u_{j} \frac{\partial u_{i} \gamma}{\partial x_{j}}+u_{j}^{r} \frac{\partial u_{i}^{r} \gamma(1-\gamma)}{\partial u_{j}^{r}}=0
$$

where $u_{j}^{r}$ is a relative velocity used to compress the interface, as documented in Berberovic et al. (2009). Any fluid property $\Phi$ in the flow is assumed to be given by

$$
\Phi=\gamma \Phi_{\text {water }}+(1-\gamma) \Phi_{\text {air }}
$$

The governing equations are solved within the open-source CFD environment OpenFOAM (version foam-extend 3.1), making use of the waves2FOAM toolbox developed by Jacobsen et al. (2012) for wave generation/absorption or specification of initial conditions. This toolbox is built upon the widely used interFoam solver, which utilizes the volume of fluid method (VOF). In both the RANS (3.1), the advection of $\gamma(3.3)$ and the turbulence model equations (2.1) and (2.2) time derivatives are discretized using a first-order implicit Euler scheme. Convection terms are discretized using a blend of central difference and upwind schemes, depending on the ratio of successive gradients. Remaining schemes are second-order accurate central difference schemes. For further details on this solver the interested reader is referred to Deshpande et al. (2012). In all forthcoming cases the time step has been adjusted such that a maximum Courant number $C o=\left|u_{i}\right| \Delta t / \Delta x_{i}=0.05$ is maintained at all times. Such a low Courant number is not typical but this ensures accurate velocity kinematics and enables nearly constant-form propagation of wave trains for long durations, as shown by Larsen et al. (2018b). Boundary conditions will be clarified on a case-by-case basis in what follows.

\subsection{Simulation of a simple progressive wave train}

In this section computed results will be presented for the long term propagation of a periodic surface wave train. This is perhaps the simplest of computational wave problems, and is a test that should ideally be passed by CFD models prior to their application on more complicated problems. As an initial condition, a numerically exact stream function wave (potential flow) solution of Fenton (1988) is specified, with no net volume flux, and with period $T=2 \mathrm{~s}$, wave height $H=0.125 \mathrm{~m}$, and water depth $h=0.4 \mathrm{~m}$ (hence $k_{w} h=0.664$ and $k_{w} H=0.207$ ). It should be noted that this wave is the same as that generated for the forthcoming simulation of the Ting \& Kirby (1994) spilling breaker experiments. Being intermediately deep and moderately nonlinear, these wave conditions are indeed well suited for generic study.

The computational domain is discretized into regular cells having horizontal and vertical size $\Delta x=\Delta z=0.01 \mathrm{~m}$ (corresponding to $H / \Delta z=12.5$ cells per wave height). This resolution has been experienced to be sufficient for the accurate propagation of this specific wave in a laminar set-up. This mesh also maintains an aspect ratio 
$\Delta x / \Delta z=1$, which can be important for accuracy, as noted by Jacobsen et al. (2012), Jacobsen et al. (2014) and Roenby et al. (2017). The computational domain spans a single wavelength, making use of periodic lateral boundaries. At the bottom boundary a slip condition is applied. This is primarily for canonical demonstration purposes, making the computational situation as close to potential flow as possible, such that a turbulence closure model should ideally not influence the physics of the wave propagation.

To demonstrate the performance of the proposed new turbulence closure relative to standard approaches, we will compare computed results from the standard Wilcox (1988) $k$ - $\omega$ model (with the buoyancy production term also included in (2.1), henceforth taken as granted) with those from our new closure with $\lambda_{1}=0$ and $\lambda_{2}=0.05$. All other model settings are kept identical. We initially set $\omega=\omega_{\infty}=2.71 \sqrt{p_{0}}$, where $p_{0}=0.66$ $\mathrm{s}^{-2}$ is the depth- and period-averaged value computed from the initial conditions, and with $k=k_{0}=\omega_{\infty} \nu$ to initially yield $\nu_{T} / \nu=1$. This is intended as a direct test of the preceding simplified analysis in an actual surface wave computation, where the standard closure should exhibit unstable behaviour from the outset, whereas the proposed new closure should remain stable indefinitely. We consider the initial condition for $\omega$ above to be most sensible in the present context, since as shown previously, it will always tend to approximately this value in the potential flow region beneath waves.

Figure 3 shows the computed temporal development of the (depth- and periodaveraged) non-dimensional eddy viscosity $\left\langle\left\langle\nu_{T}\right\rangle\right\rangle / \nu$ with both closure models considered. Included in the figure (dashed line) is also the predicted development of $\left\langle\left\langle\nu_{T}\right\rangle\right\rangle / \nu=$ $\exp \left(\Gamma_{\infty} t\right)$ with the growth rate from (2.22). Figure 3 clearly shows that the Wilcox (1988) $k$ - $\omega$ model is indeed initially unstable, resulting in an exponentially growing eddy viscosity (hence also $k$ ) that ultimately becomes several orders of magnitude larger than the kinematic viscosity, before eventually levelling off. The computed exponential growth rate is near that expected based on the simplified analysis, which has neglected several terms. It is emphasized that the growth of turbulence does not come from the bottom boundary layer, which is non-existent as a slip condition has again been used. Nor does it stem from the free-surface, where the buoyancy production term in equation (2.7) suppresses turbulent kinetic energy in this region. Rather, it is due to the instability of the potential flow region, as confirmed via the close match with the theoretical growth rate. In contrast, also consistent with the preceding analysis, our new modified closure remains stable, with the eddy viscosity quickly decaying to physically insignificant levels.

The eventual levelling off of the eddy viscosity with the standard $k-\omega$ of Wilcox (1988) can be explained as due to declining wave heights, which are a direct consequence of the un-physical growth of the turbulent kinetic energy and eddy viscosity. These eventually reach levels that are sufficiently high to cause un-physical turbulent diffusion of the wave, with energy thus being extracted from the mean flow. As a result of the decreased wave height the turbulence production quantity $p_{0}$ is likewise reduced. This can be seen from the surface elevation time series depicted in figure 4(a), where the waves begin to decline after only $t \approx 20 T$. The decay in wave height is similar to what was shown by Mayer \& Madsen (2000) and Devolder et al. (2017). In contrast, the wave evolution computed with the new stabilized closure maintains a nearly constant wave height, as seen from figure 4(b). The slight decay seen is clearly due to minor numerical diffusion associated with the numerical scheme, and not in any way related to the new turbulence closure, as it has effectively switched itself off. This is clear from the previously-mentioned extremely small eddy viscosity in figure 3, as well as through comparison with an additional (otherwise identical) simulation with the turbulence model switched off entirely, which results in visually identical behaviour (not directly shown here for the sake of brevity). Note that 


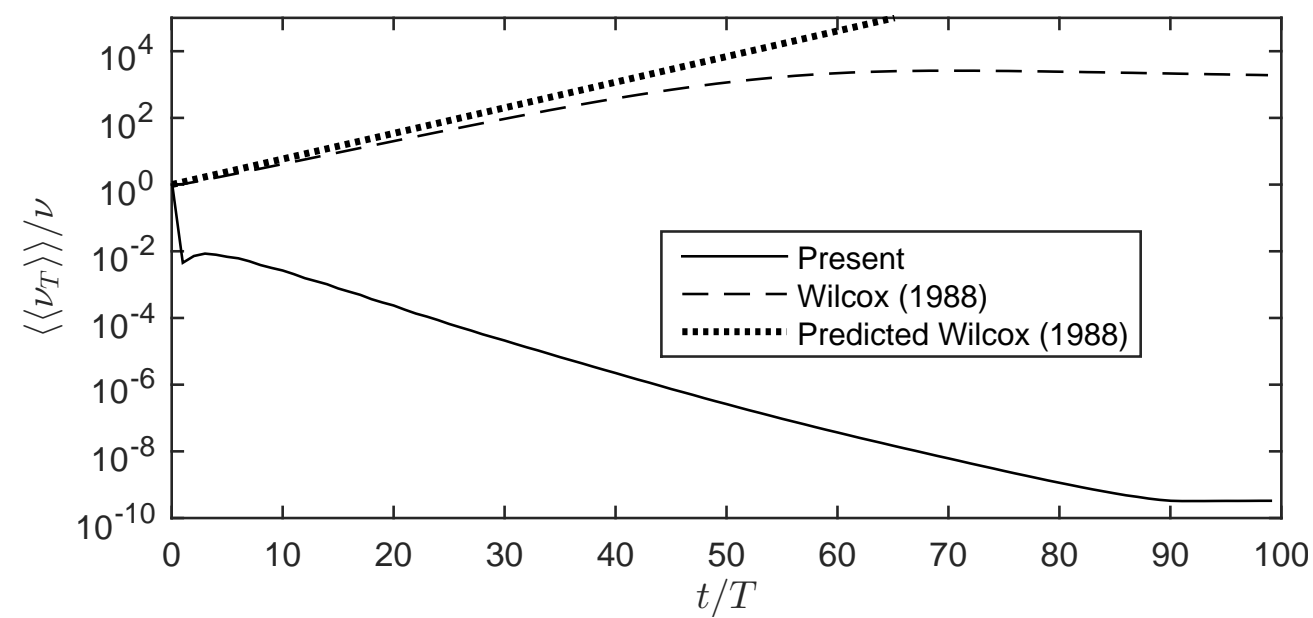

Figure 3. Computed temporal development of the depth- and period-averaged eddy viscosity beneath a periodic wave train using the present $k-\omega$ closure model with $\lambda_{1}=0$ and $\lambda_{2}=0.05$ (full line), the Wilcox (1988) $k$ - $\omega$ model (dashed line), as well as the predicted development from (2.22) (dotted line).
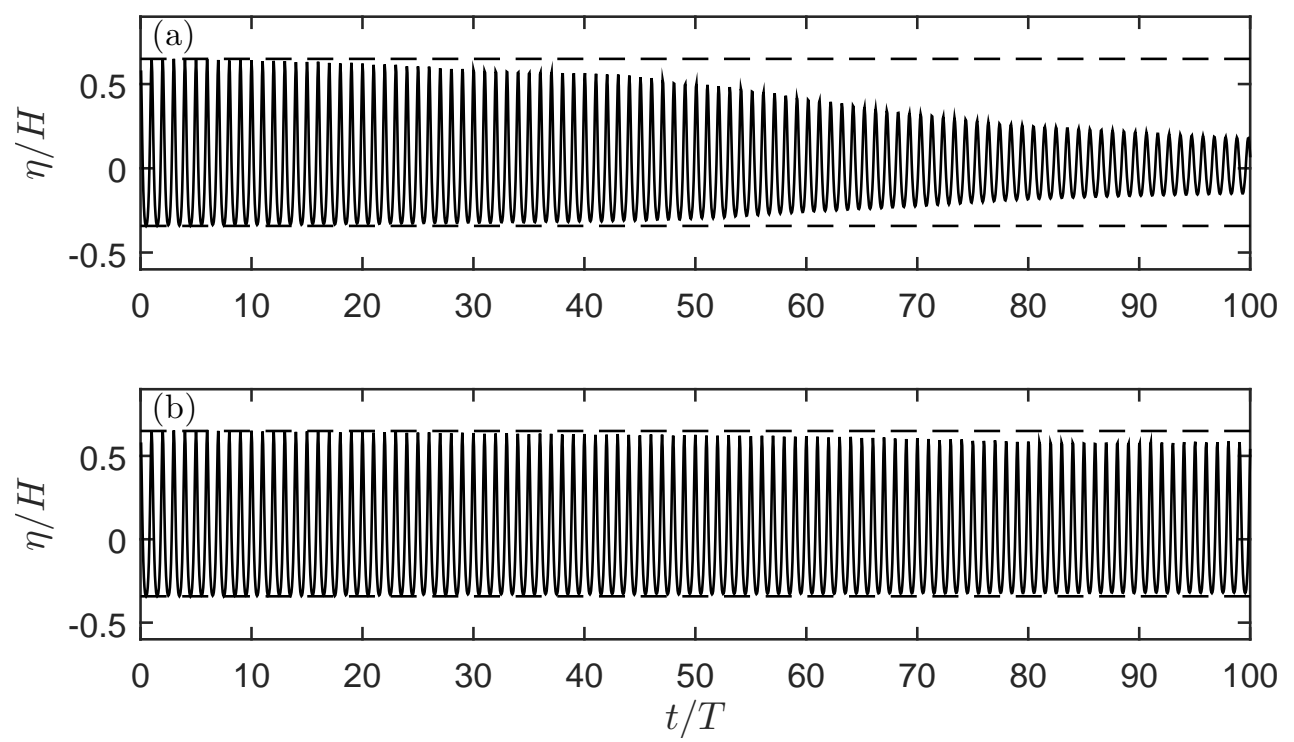

FiguRE 4. Computed time series of surface elevations in the simulation of a periodic wave train using (a) the standard Wilcox (1988) $k$ - $\omega$ model and (b) the proposed new closure model with $\lambda_{1}=0$ and $\lambda_{2}=0.05$. The horizontal dashed lines represent the crest and trough levels of the initial wave, for comparison.

we have also made other, similar, simulations as above, with $0.02 \leqslant \lambda_{2} \leqslant 0.1$, which result in similar surface elevations as in figure $4(\mathrm{~b})$.

This example demonstrates how the instability identified by Mayer \& Madsen (2000), and which is inherent in standard two-equation turbulence closure models as shown herein, can manifest in their rather spectacular failure in even the simplest of surface wave computations. The comparison above likewise demonstrates that such failure can be avoided entirely by employing the simple new (stabilized) closure proposed herein. 


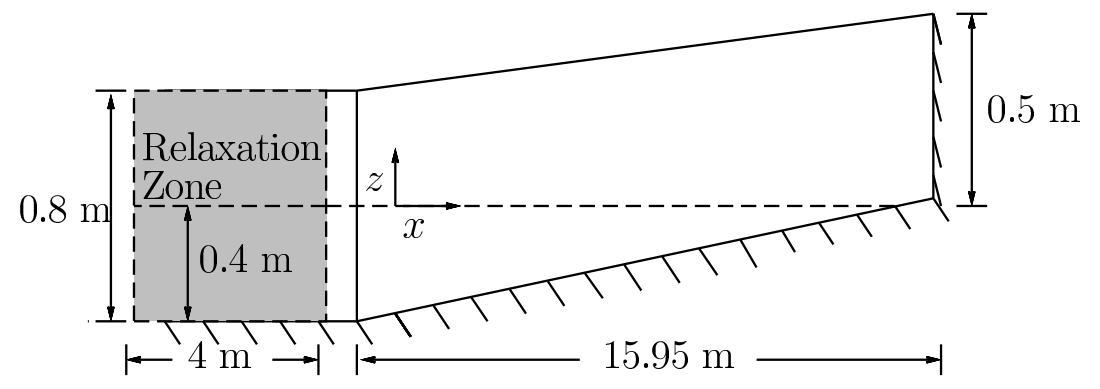

FiguRE 5. Layout of the computational domain for the simulation of the spilling breaking wave experiments of Ting \& Kirby (1994).

\subsection{Simulation of spilling breaking waves}

As a follow-up to the preceding example, let us now consider simulations of the spilling breaking wave experiment of Ting \& Kirby (1994), to demonstrate the performance of the new model in a more physically complex situation. We focus here exclusively on their spilling, rather than plunging, case as it is these incoming wave conditions where the over-production of turbulence has consistently been observed in prior studies. Quantitatively the reason for this can be explained by equation (2.13) which yields $\left\langle\left\langle p_{0}\right\rangle\right\rangle=0.55 \mathrm{~s}^{-2}$ and $\left\langle\left\langle p_{0}\right\rangle\right\rangle=0.08 \mathrm{~s}^{-2}$ for their spilling and plunging cases respectively, i.e. a much lower turbulence production and hence unstable growth rate for the plunging case. It is emphasized that the strength of the instability does not strictly depend on the breaker type (which also depends on the slope encountered), but rather only on the characteristics of the incoming waves. (Note that the interested reader can find results from similar simulations utilizing our stabilized closure on the plunging breaking case in the PhD thesis of Larsen 2018).

The model domain for these simulations consists of a flat region having water depth $h=0.4 \mathrm{~m}$, connected to a region having constant 1:35 slope. For these simulations, the same waves as considered previously $(T=2 \mathrm{~s}$ and $H=0.125 \mathrm{~m}$ with zero net volume flux; $k_{w} h=0.664$ and $\left.k_{w} H=0.207\right)$ are generated on the horizontal bed. At the left inlet boundary a relaxation zone of $4 \mathrm{~m}$ is used, which serves to absorb any waves reflected by the slope, thus the incoming waves do not change over time. In figure 5 a layout of the computational domain is seen. For the purposes of consistent comparison, the origin is positioned at the same depth $(h=0.38 \mathrm{~m})$ as in the experiments. No roughness was indicated in the experiment, but it was stated that the bed was made of plywood, and therefore the model bed is assigned a Nikuradse equivalent sand roughness $k_{s}=10^{-4} \mathrm{~m}$.

The initial condition for $\omega$ is again taken as $\omega=\omega_{\infty}=2.71 \sqrt{p_{0}}$, with $p_{0}$ calculated from (2.13) and with $k=k_{0}=0.1 \omega_{\infty} \nu$ such that $\nu_{T} / \nu=0.1$ (this is one order of magnitude below that used in the previous progressive wave train test case in §3.2). In contrast to the previous idealized simulation in $§ 3.2$, a no-slip boundary condition is imposed at the bottom, meaning that a wave boundary layer will now develop near the bed, as in reality. Turbulence quantities in the first cell near the bottom are prescribed using the generalized wall functions presented in Fuhrman et al. (2014), which build upon the generalized van Driest profile of Cebeci \& Chang (1978). These wall functions have been used succesfully in the simulation of various scour processes, see e.g. Baykal et al. (2015), Bayraktar et al. (2016), Larsen et al. (2016), Larsen et al. (2017) and Larsen et al. $(2018 a)$, and allow for near-bed cells to lie in either the logarithmic or viscous sub-layer. On the flat region the domain is discretized into cells with $\Delta x=\Delta z=0.01 \mathrm{~m}$, and on the slope the mesh is gradually refined towards the shore while keeping a constant aspect 


\begin{tabular}{ccccccc}
\hline Case & $k$ - $\omega$ model & $\lambda_{1}$ & $\lambda_{2}$ & $\tilde{\tilde{\omega}}$ & $\tilde{\omega}$ & Line type (Figs. 12 \& 13) \\
\hline 1 & Wilcox (1988) & 0 & 0 & $=\omega$ & $=\omega$ & Dashed light (blue) line \\
2 & Wilcox (2006) & 0.875 & 0 & $=\tilde{\omega}$ & Eq. (2.29) & Dotted light (blue) line \\
3 & Present & 0 & 0.05 & $=\omega$ & Eq. (2.35) & Dashed dark (black) line \\
4 & Present & 0.875 & 0.05 & Eq. (2.34) & Eq. (2.35) & Dotted dark (black) line \\
5 & Present & 0.2 & 0.05 & Eq. (2.34) & Eq. (2.35) & Solid dark (black) line \\
\hline
\end{tabular}

TABLE 2. Summary of parameters used in the five different simulations of the Ting \& Kirby (1994) spilling breaking wave experiment.

ratio of unity. At the right end of the domain the cells have a size $\Delta x=\Delta z=0.0063 \mathrm{~m}$. Near the bed layers of cells are refined in the vertical direction with near bed cells having height $\Delta z=7.5 \cdot 10^{-4} \mathrm{~m}$. This ensures that $\Delta z^{+}=\Delta z U_{f} / \nu<30$ during the simulation, with $U_{f}$ being the friction velocity.

For the sake of systematic comparison, simulations of these experiments will be considered using five different turbulence closure variants. These correspond to: (Case 1) the standard Wilcox (1988) $k$ - $\omega$ model, (Case 2) the Wilcox (2006) $k$ - $\omega$ model, as well as three variants of the proposed modified $k-\omega$ closure with fixed $\lambda_{2}=0.05$ and: (Case 3) $\lambda_{1}=0$, (Case 4) $\lambda_{1}=0.875$, and (Case 5) $\lambda_{1}=0.2$. The $\lambda_{1}$ values in Cases 3 and 4 correspond to those associated with the models of Wilcox (1988) and Wilcox (2006) (thus allowing direct comparison) i.e. Cases 3 and 4 correspond to stabilized versions of Cases 1 and 2, respectively. Case 5 utilizes an intermediate value for $\lambda_{1}$, more in line with Eq. (9) of Durbin (2009) (corresponding to $\lambda_{1}=0.26$ ). For ultimate clarity, the parameter settings used in these five cases are listed in table 2 . We shall henceforth refer to these simulations by their case number, as indicated in table 2 and in the text immediately above.

Figure 6 shows computed snapshots depicting the surface and non-dimensional eddy viscosity $\nu_{T} / \nu$ from each of the five model variants mentioned above, following a long simulation time of 100 periods, such that steady (repeatable) conditions have effectively been reached. (As an indication of simulation time, each run requires approximately three weeks when simulated in parallel on eight modern processors.) Each plot is frozen at an instant where the wave is very close to breaking, such that the location of the surf zone is evident (beginning approximately at $x=x_{b}=6.4 \mathrm{~m}$ ). As can be seen, both the standard Wilcox (1988) (Case 1, figure 6(a)) and the Wilcox (2006) $k$ - $\omega$ models (Case 2, figure 6(b)) result in uniformly high eddy viscosity (orders of magnitude larger than $\nu$ ), even in the pre-breaking region. These high values are in no way physical, as in real waves significant turbulence should not be expected prior to breaking, see e.g. the measured turbulence levels from Ting \& Kirby (1994) and van der A et al. (2017), the PIV experiments of Chang \& Liu (1998), Kimmoun \& Branger (2007), Belden \& Techet (2011), and Grue \& Jensen (2006) or the dye experiment of Duncan et al. (1999). Rather, these high values are again an artefact related to the inherent instability of these models in the nearly-potential flow regions which are prevalent leading up to the surf zone. Conversely, regardless of the value of $\lambda_{1}$, the three results using the proposed new stabilized closure (figures $6(\mathrm{c})-(\mathrm{e})$ corresponding to Cases 3-5) predict negligible eddy viscosity in the bulk region beneath the waves prior to breaking; Significant eddy viscosity with these stabilized models is rightly confined to the near bed boundary layer and surf zone, demonstrating a clear 

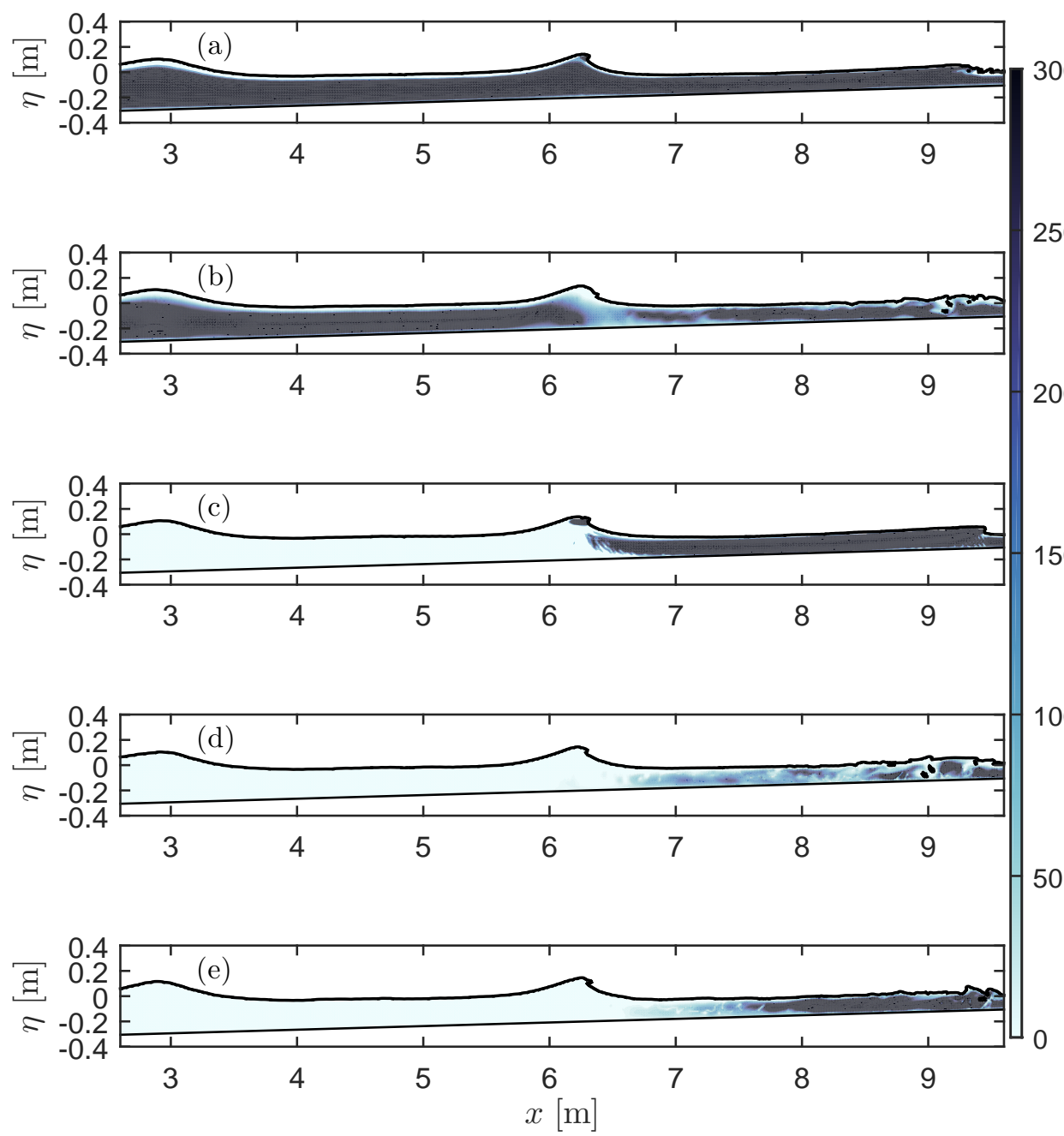

Figure 6 . Snapshots of $\nu_{T} / \nu$ at $t / T=100$ with the wave right at breaking computed with the (a) standard Wilcox (1988) $k$ - $\omega$ model (Case 1), (b) Wilcox (2006) $k$ - $\omega$ model (Case 2), and (c)-(e) present stabilized $k-\omega$ closure model (respectively, Cases 3-5), where parameters for each case are indicated in table 2 . The free surface and bottom are marked by a solid line.

and qualitative correction over existing models. Figure 6 is hence a clear demonstration of how, due to their inherent instability, existing standard turbulence closure models in wide use can result in severely polluted results prior to wave breaking, often the very phenomenon of interest in CFD studies where such models are employed. This figure likewise definitively demonstrates that the new stabilized closure model proposed herein eliminates this problem altogether.

To provide further details on the computed vorticity-to-strain ratios found in the present simulations, figure 7 depicts $\left\langle p_{\Omega}\right\rangle /\left\langle p_{0}\right\rangle$ beneath trough level, averaged over 


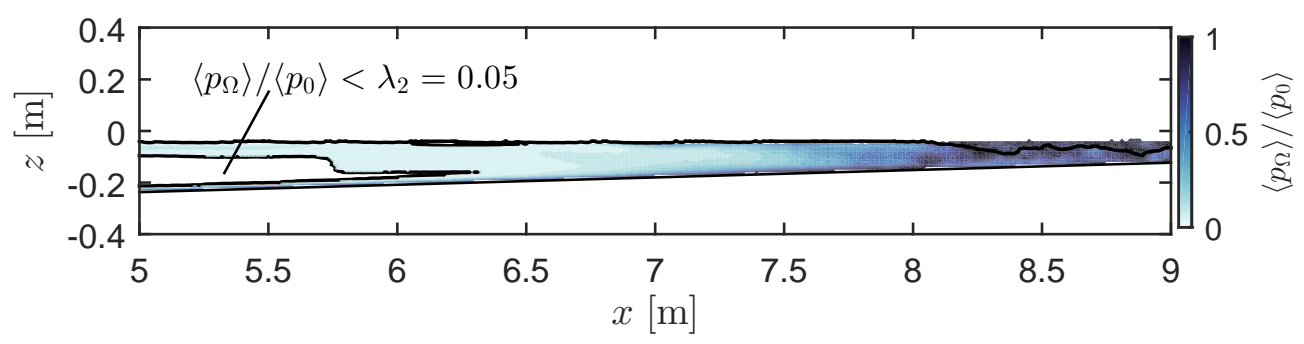

Figure 7. Computed $\left\langle p_{\Omega}\right\rangle /\left\langle p_{0}\right\rangle$ below trough level for Case 5. The region where $\left\langle p_{\Omega}\right\rangle /\left\langle p_{0}\right\rangle<\lambda_{2}=0.05$ is indicated in white.

$60<t / T<120$ for Case 5 (as an example of our stabilized approach). The color scheme in this figure has been chosen such that the white region corresponds to nearly-potential flow i.e. where $\left\langle p_{\Omega}\right\rangle /\left\langle p_{0}\right\rangle\left\langle\lambda_{2}=0.05\right.$, and the new stabilizing limiter would thus be (on average) active. Conversely, colored (shaded) regions correspond to sheared flow where this threshold value is exceeded, and the new limiter would essentially be off. It is seen that the location where the nearly-potential flow region ends closely coincides to the beginning of the surf zone (again, beginning approximately at $x=x_{b}=6.4 \mathrm{~m}$ ), as should be expected. Prior to breaking the proposed limiter is typically active only in the nearly-potential flow core region in the middle of the water column, whereas it is inactive in the sheared regions below and above, respectively corresponding to boundary layer regions which form where the water interacts with the bed and air (note that $\left\langle p_{\Omega}\right\rangle$ and $\left\langle p_{0}\right\rangle$ are the same order of magnitude in these regions). This figure also clearly shows that in the surf zone $\left\langle p_{\Omega}\right\rangle$ and $\left\langle p_{0}\right\rangle$ are generally within an order of magnitude of one another, though they are often far from equal. In the inner surf zone they are even more similar and are typically within a factor of two. This figure, in conjunction with the eddy viscosity plots in figure 6 , thus further confirms that (as designed) the new limiter is primarily only active in the nearly-potential flow region prior to breaking, while essentially remaining passive in the surf zone and other sheared regions.

We will now further demonstrate the differences between results computed with standard (unstable) and new closure models via further quantitative comparison against the spilling breaker data set of Ting \& Kirby (1994). To make proper comparisons, as in the experiments, a relatively long warm up period is needed to establish stable conditions in the computational flume. Before extracting data, models have therefore been run for 60 periods. Such a long warm up period has not been common in most previous numerical studies of the Ting \& Kirby (1994) experiments. Jacobsen et al. (2012) demonstrated, however, that at least 40 periods were necessary in order to reach a constant volume of water in their domain. We have similarly found that an additional 20 periods were necessary to achieve a convincing quasi-steady situation. For comparison, it can be noted that the warm up length in the experiments was 600 periods. Furthermore, in order to achieve stable mean values, the results presented in the following have been obtained by averaging over an additional 60 periods following the warm up (i.e. simulations have been run for a total of 120 periods). For comparison, in the original experiments the results were averaged over a comparable duration corresponding to 102 periods.

Figure 8 shows comparison of the computed and experimental surface elevation envelopes as well as the mean water levels for the five models mentioned above. The solid lines represent the mean (ensemble averaged) values, whereas the shaded area represents 
plus or minus one standard deviation, to give an indication of wave-to-wave variability. It can be seen that all five models, in general, capture the evolution of the mean surface elevations well. The horizontal position of the breaking point and surface elevation at breaking is well captured, as is the subsequent decay in wave heights in the surf zone. These results compare well with surface elevations presented in other numerical studies of these experiments, see e.g. Jacobsen et al. (2012), Hieu et al. (2004), Xie (2013) and Brown et al. (2016). The most notable difference between the models is the standard deviation in the surface elevations in the surf zone. As seen, those results sharing $\lambda_{1}=0$ (i.e. the Wilcox (1988) model, Case 1 shown in figure 8(a) and our stabilized version of this model, Case 3 shown in figure 8(c)) demonstrate little wave-to-wave variability. Conversely, the three results with the Wilcox (2006) limiter active $\left(\lambda_{1}>0\right.$, Cases 2 , 4 and 5, respectively depicted in figure $8(\mathrm{~b}),(\mathrm{d}),(\mathrm{e}))$ result in significant wave-to-wave variability during the breaking process. Such variability is much more in line with the experiments (see e.g. figure 3 of Ting \& Kirby 1994).

Further inspection has revealed that this lack in wave-to-wave variability computed with $\lambda_{1}=0$ is due to the waves not breaking properly. This is due to large eddy viscosity computed in the crest (see figure $6(\mathrm{a}),(\mathrm{c})$ ), leading to turbulent dissipation of the wave rather than a convincing sequence of spilling breaking. This is illustrated in figure 9, where snapshots of typical waves computed during the breaking process are compared for Cases $3\left(\lambda_{1}=0\right)$ and $4\left(\lambda_{1}=0.875\right)$ i.e. two variants of the present stabilized model having different $\lambda_{1}$, but being otherwise identical. As a result of this failure of the waves to properly spill with $\lambda_{1}=0$, there is little deviation in the surface elevations experienced in successive waves in the surf zone. In contrast, the cases computed with $\lambda_{1}>0$, figure $8(\mathrm{~b}),(\mathrm{d}),(\mathrm{e})$, spilling breaking occurs, leading to a much more dynamic surf zone. These results indicate that using a Wilcox (2006)-type limiter in the turbulent production is necessary to qualitatively capture the spilling breaking process. In the authors' opinion, while at first glance the five results in figure 8 may appear similar, the inability of the $k$ - $\omega$ models with $\lambda_{1}=0$ (figure 8(a),(c) corresponding to Cases 1 and 3) to predict proper spilling breakers (and corresponding wave-to-wave variability) can be regarded as an important qualitative shortcoming. We emphasize that the features discussed above (nature of breaking and subsequent wave-to-wave variability) are primarily dependent on the Wilcox (2006)-type limiter $\left(\lambda_{1}\right)$, and have little to do with the new stabilizing limiter $\left(\lambda_{2}\right)$ introduced in $(2.35)$.

To further illustrate the difference between cases with $\lambda_{1}=0$ and $\lambda_{1}>0$, figure 10 shows comparison between the experimental and computed phase-averaged surface elevations for Case 3 and 5 at four different post-breaking cross shore positions. In general the phase-averaged surface elevations match well for both cases, but there are two noteworthy features. First, in figure 10(a)-(c) the increase in surface elevation in Case 3 is more rapid than in Case 5 . This can again be explained by the wave not properly breaking in Case 3 at these positions (as just shown in figure 9) resulting in a maintained steeper wave front. Second, in figure 10(d) Case 5 is significantly leading the experiments, indicating that the surface roller is apparently travelling too fast in the inner surf zone. The implications of this on the wave kinematics will be discussed later in this section.

Returning back to figure 8 , considering the previous example from $\S 3.2$, it may be somewhat surprising that the Wilcox (1988) and Wilcox (2006) models perform at all reasonably in terms of the mean surface elevations (figure $8(\mathrm{a}),(\mathrm{b})$ ). One might have expected that the large eddy viscosity would cause the waves to decay even prior to breaking, as was seen in the previous demonstration involving a propagating wave train (figure 4(a)). This is, in fact, a real possibility, and the reason why this did not occur in the previous examples is merely due to the short propagation distance (in this case only 

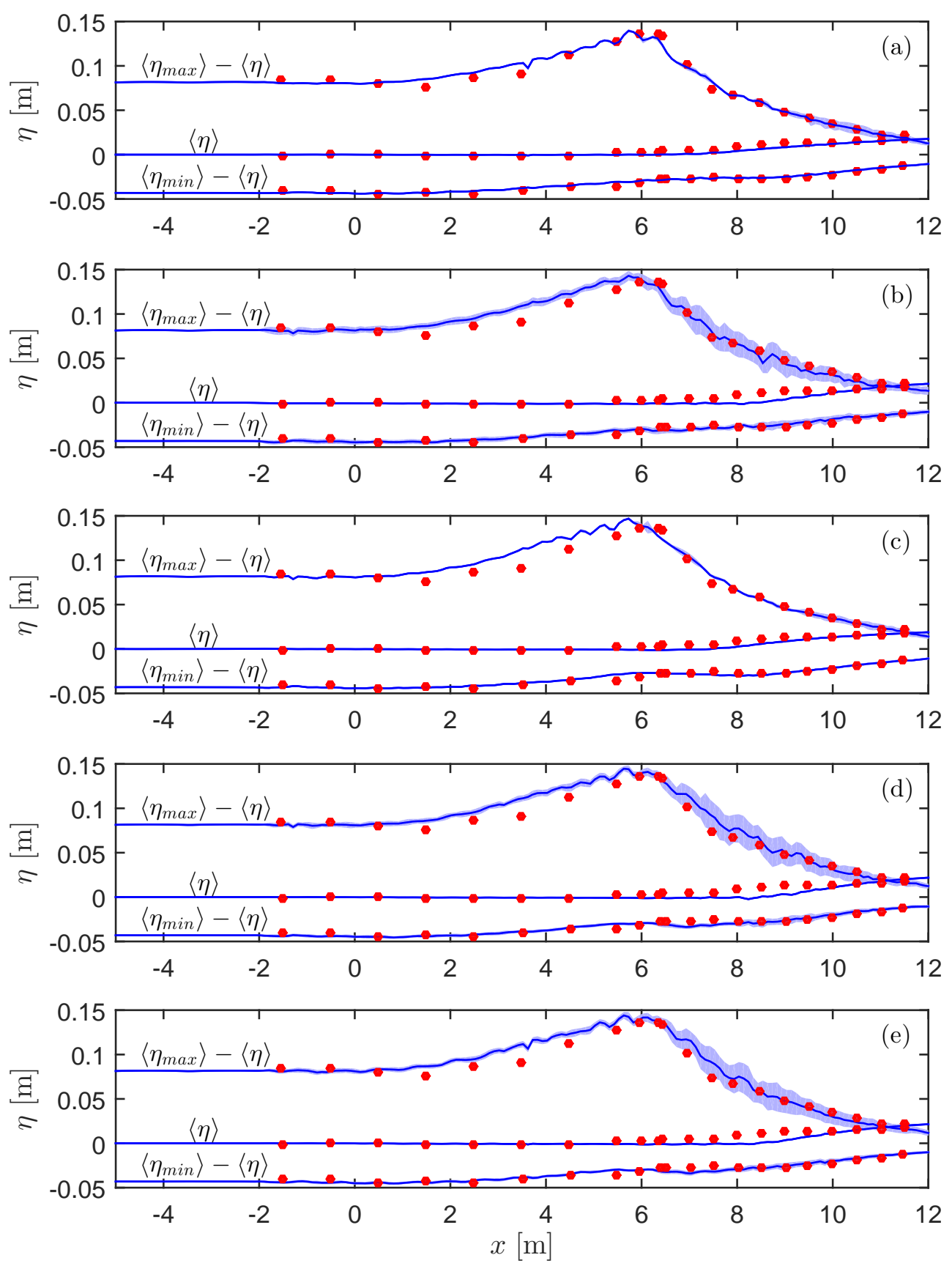

Figure 8. Comparison of modelled (lines) and measured (circles, from Ting \& Kirby 1994) surface elevation envelopes (top and bottom lines) and mean water levels (middle lines). Results in (a)-(e) correspond to Cases 1-5, respectively. Solid lines represents the mean surface elevation and the shaded area represents plus and minus one standard deviation. 

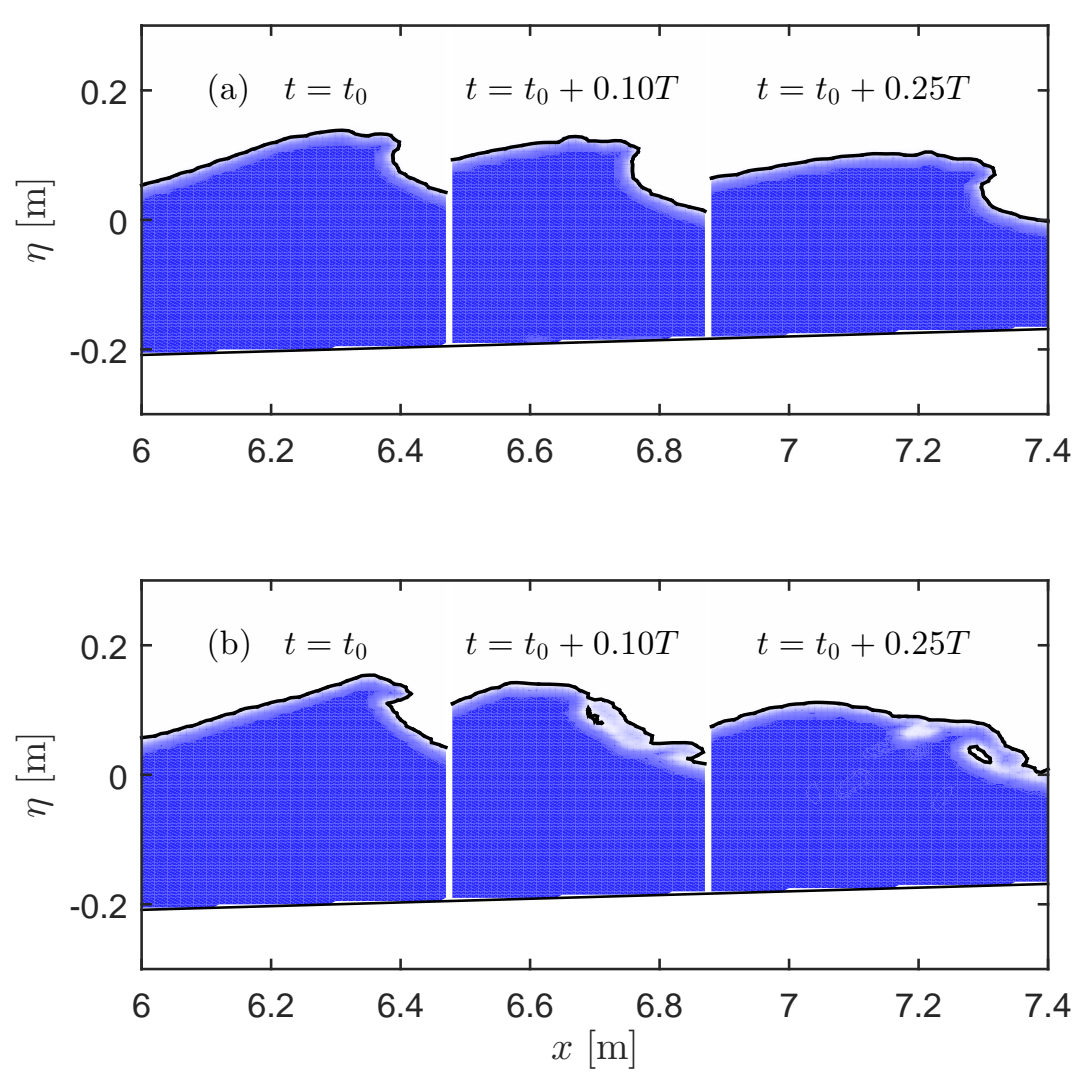

FIGURE 9. Snapshots of the breaking process computed in (a) Case $3\left(\lambda_{1}=0, \lambda_{2}=0.05\right)$ and (b) Case $4\left(\lambda_{1}=0.875, \lambda_{2}=0.05\right)$, beginning at $t=t_{0}=110 T$. Here dark represents water, white represents air, and light shades represent air-water mixtures. The wave shown in (a) never actually spills.

a few wave lengths) allotted prior to the onset of breaking, which limits the extent of the turbulence over-production problem. Such pre-breaking wave decay would most certainly occur if the pre-slope distance was extended sufficiently further. To show this we have performed one additional simulation, but with the flat region now being $40 \mathrm{~m}$ instead of $4.7 \mathrm{~m}$. The resulting surface elevations using the Wilcox (1988) model are shown in figure 11. Here it can be seen that the unstable growth in the eddy viscosity has caused the incoming wave to decline significantly, and as a consequence the horizontal position of the breaking point has shifted to be further onshore. Importantly, this recognition implies that previously computed results of breaking waves with non-stabilized closure models (prevalent in the literature) are not unique, instead being heavily dependent on both the initial conditions and the allotted propagation distance prior to shoaling and breaking. Results computed with the new stabilized closure, on the other hand, will be fundamentally insensitive to these issues.

As further comparison, figure 12 presents computed and measured (Ting \& Kirby 1994) averaged turbulent kinetic energy $k$ profiles at a total of eight positions, corresponding to two pre-breaking positions (figure 12(a),(b)), as well as six in the surf zone (figure $12(\mathrm{c})-(\mathrm{h}))$. In the experiments only two components of the velocity were measured, so $k$ 

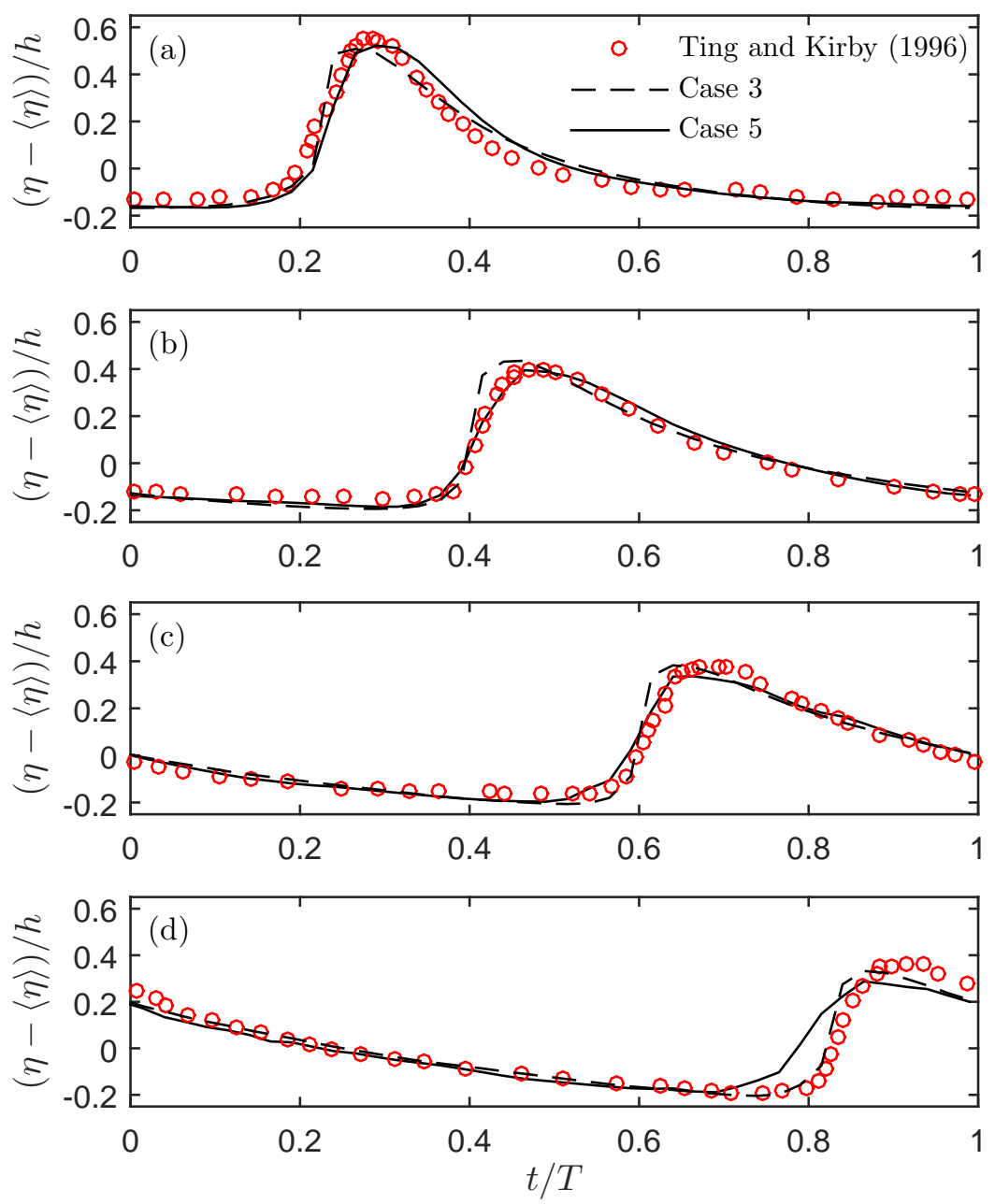

Figure 10. Comparison of modelled (lines) and measured (circles) phase-averaged surface elevations at (a) $x=7.275 \mathrm{~m}\left(\tilde{x}=\left(x-x_{b}\right) / h_{b}=4.397\right)$, (b) $x=7.885 \mathrm{~m}(\tilde{x}=7.462)$, (c) $x=8.495 \mathrm{~m}(\tilde{x}=10.528)$, and $(\mathrm{d}) x=9.11 \mathrm{~m}(\tilde{x}=13.618)$

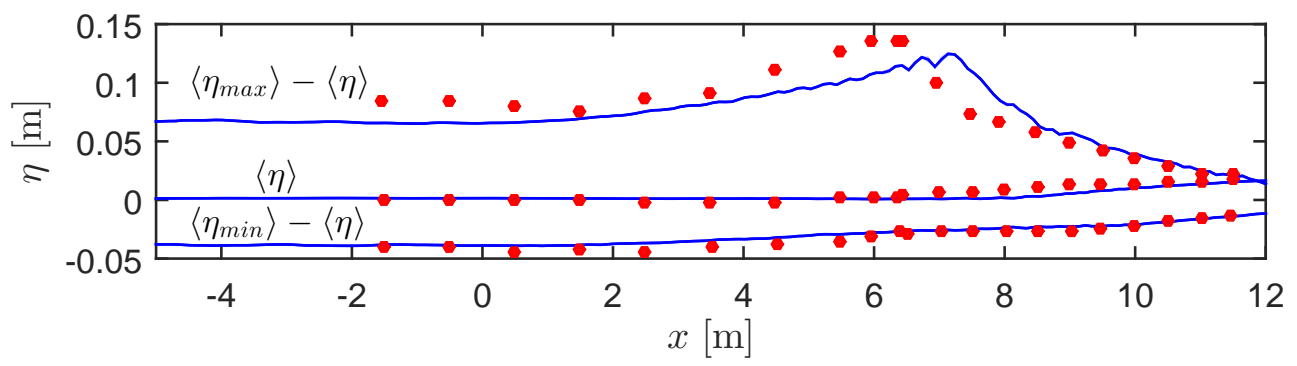

Figure 11. Comparison of computed (lines) and measured (Ting \& Kirby 1994, circles) surface elevation envelopes and mean water levels using the Wilcox (1988) model with a long (40 m) flat region. This plot can be compared directly with figure 8(a), which depicts results computed with the same model, but where the flat region is only $4.7 \mathrm{~m}$. 
was approximated by $k \approx 1.33 / 2\left(\overline{u^{\prime 2}}+\overline{w^{\prime 2}}\right)$. This approximation was also used by Stive $\&$ Wind (1982) and Svendsen (1987), and comes from the results of a plane wake from Townsend (1976). However more recent results from Scott et al. (2005) indicate that

$$
k \approx 0.75\left(\overline{u^{\prime 2}}+\overline{w^{\prime 2}}\right)
$$

in the surf zone, and this approximation has been used in what follows. Ting \& Kirby (1994) did not provide approximate $k$-profiles in the two pre-breaking positions and the first post-breaking position (figure 12(a)-(c)) as well as the top three measurement positions, but did provide profiles of the single component $\overline{u^{\prime 2}}$. At these positions we have alternatively utilized the approximation

$$
k \approx 1.25 \overline{u^{\prime 2}}
$$

again based on the measurements of Scott et al. (2005). It is thus emphasized that the experimental $k$-profiles must all be regarded as approximate, but are still likely reasonably indicative. For further discussions or indications on the uncertainties of such approximations see e.g. Scott et al. (2005) and Ting \& Kirby (1996).

To ease comparison the computed results in figure 12 are organized such that results with light shaded (blue) lines correspond to non-stabilized closures $\left(\lambda_{2}=0\right.$, Cases 1 and 2 ), whereas dark (black) lines correspond to formally stabilized models $\left(\lambda_{2}=0.05\right.$, Cases $3-5)$. Moreover, results having the same line type share common $\lambda_{1}$ values, see again table 2. As might by now be expected, the standard Wilcox (1988) (Case 1) and Wilcox (2006) (Case 2) $k$ - $\omega$ models severely over-predict the turbulence pre-breaking (light shaded/blue lines in figure 12(a),(b)), which may again be regarded as a direct consequence of the instability of these models. The depicted over-production of turbulence using the Wilcox (1988) and Wilcox (2006) models is typical of previous studies. In contrast, the new stabilized closure models (Cases 3-5) predict low turbulent kinetic energy pre-breaking (dark black lines in figure 12(a),(b), see also again figure 6), much more in line with the experiments. The improvement seen with the new stabilized closures in figure 12(a),(b), and related effects further shoreward, can be regarded as the principal achievement of the present work: Only waves computed with the new stabilized closure arrive at the surf zone, and hence begin the simulated breaking process, un-polluted. To most clearly see the isolated effect of the stabilizing limiter $\lambda_{2}$, Cases 1 and 2 can be respectively compared with Cases 3 and 4 .

The over-production of turbulence prior to breaking using the non-stabilized models also has an effect on the post-breaking turbulence. As can be seen, both the Wilcox (1988) and Wilcox (2006) models (Cases 1 and 2) predict higher turbulence levels than their stabilized counterparts (respectively, Cases 3 and 4) during the initial breaking process (figure 12(c)-(e)). This demonstrates that the un-physically high levels of pre-breaking turbulence predicted by the non-stabilized models can indeed pollute results extending well into the outer surf zone. Once the inner surf zone is reached (figure $12(\mathrm{f})-(\mathrm{h})$ ) the differences between the stabilized (dark black lines) and standard (light shaded/blue lines) turbulence models become smaller. Rather, the results in the inner surf zone are more governed by the value of the Wilcox (2006) stress limiter coefficient $\lambda_{1}$ and have relatively little to do with $\lambda_{2}$. This can be clearly seen in figure 12 , where the results having the same line type i.e. Cases 1 and 3 (dashed lines), and Cases 2 and 4 (dotted lines), which were initially quite different in figure $12(\mathrm{a})-(\mathrm{c})$, have become quite similar in figure 12(f)-(h). Thus, the stabilization of the closure models achieved by utilizing $\lambda_{2}>0$, as introduced herein, plays an important role both prior to breaking and in the outer surf zone, while expectedly becoming less important in the inner surf zone. This behaviour is as intended, as it has again been the aim of the present work to produce 

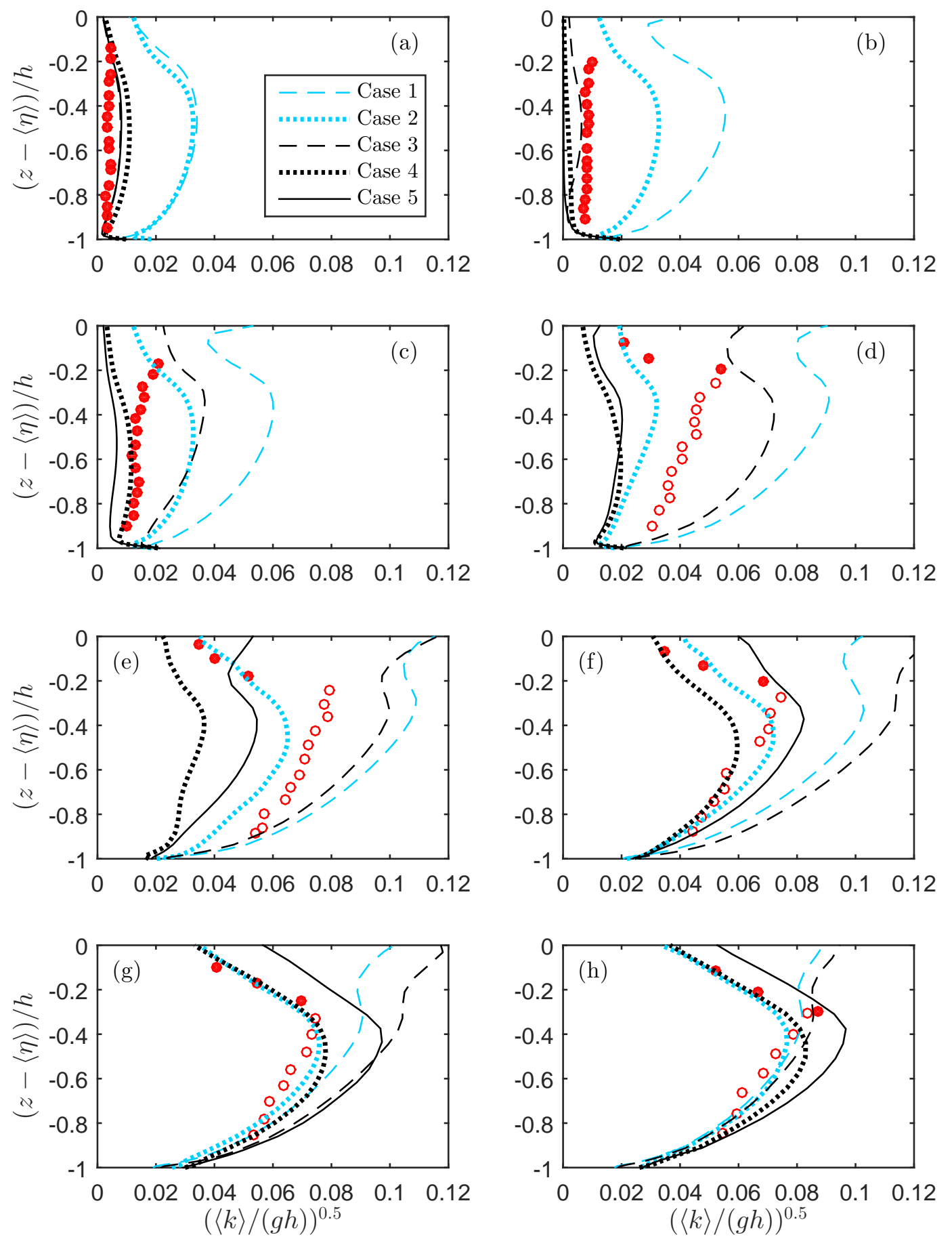

Figure 12. Comparison of modelled (lines) and measured (empty circles: from (3.5), filled circles: from (3.6)) turbulent kinetic energy $k$ profiles at (a) $x=-1.265 \mathrm{~m}$ $\left(\tilde{x}=\left(x-x_{b}\right) / h_{b}=-38.518\right)$, (b) $x=5.945 \mathrm{~m}(\tilde{x}=-2.286)$, (c) $x=6.665 \mathrm{~m}(\tilde{x}=1.332)$, (d) $x=7.275 \mathrm{~m}(\tilde{x}=4.397)$, (e) $x=7.885 \mathrm{~m}(\tilde{x}=7.462)$, (f) $x=8.495 \mathrm{~m}(\tilde{x}=10.528)$, (g) $x=9.11 \mathrm{~m}(\tilde{x}=13.618)$ and $(\mathrm{h}) x=9.725 \mathrm{~m}(\tilde{x}=16.709)$. 
a formally stabilized closure model in potential flow regions, which defaults to a desired existing closure in sheared regions (i.e. the surf zone in the present context). The results in figure 12, as a whole, demonstrate that this has indeed been achieved.

To demonstrate the consequences directly on the flow properties, the computed and measured averaged undertow velocity profiles $\langle u(z)\rangle$ are finally compared in figure 13, at the same eight positions as in figure 12. Figure 13(a),(b) clearly illustrates the adverse effects associated with the pre-breaking over-production of turbulence inherent within the standard Wilcox (1988) and Wilcox (2006) models (Cases 1 and 2, respectively). The negative peaks in the computed undertow with these models are consistently near the sea bed, whereas the measurements show these to be much higher up. This qualitative difference is important and can be explained by the artificially high $\nu_{T}$ in the upper part of the wave increasing the flow resistance, thus resulting in the strongest undertow near the bottom (similar to those profiles measured deeper into the surf zone). In contrast, in the experiments, as well as with the proposed new stabilized closure models (dark lines, Cases 3-5), the flow resistance is largest near the bed at these positions, and hence the undertow is strongest in the upper part of the flow.

The effects of over-produced turbulence in the outer surf zone are also clearly visible, with the two models having $\lambda_{1}=0$ (Cases 1 and 3, dashed lines) maintaining an erroneous undertow structure (figure 13(c),(d)) while the cases with $\lambda_{1}>0$ (Cases 2, 4 and 5) show a better evolution of the undertow structure at these positions. These results thus, again, highlight the positive influence of the Wilcox (2006) stress limiter, which was previously found essential to obtain properly spilling waves (figure 9). The model which best matches the evolution of the measured undertow structure from pre-breaking and throughout the outer surf zone (figure $13(\mathrm{a})-(\mathrm{e})$, corresponding to $-1.265 \mathrm{~m} \leqslant x \leqslant 7.885 \mathrm{~m}$ ) is Case 5 , corresponding to the present stabilized $\left(\lambda_{2}=0.05\right)$ closure with the intermediate value $\lambda_{1}=0.2$. Notably, this transition from shoaling to the outer surf zone is a physically important and complex region of great interest, as the physics associated with the pre-topost breaking transformation are related e.g. to the formation and dynamics of near-shore breaker bars, which play an important role in coastal protection.

Consistent with the trends seen previously in the computed turbulent kinetic energy, the results in the inner surf zone become grouped largely based on the value of $\lambda_{1}$ utilized (figure 13(f)-(h)) i.e. the results using (otherwise identical) formally stabilized or nonstabilized models are essentially similar in this region. The velocity profiles computed with $\lambda_{1}>0$ (Cases 2, 4 and 5) remain qualitatively correct in structure, but become exaggerated by a factor of approximately two relative to those measured. It can be noted that very similar results for the undertow in the inner surf zone have been shown with various RANS closure models in Brown et al. (2016), as well as with a LES model in Christensen (2006). In contrast, the models using $\lambda_{1}=0$ (Cases 1 and 3, dashed lines) actually result in more accurate undertow profiles in the inner surf zone. These results are certainly interesting, though in the authors' opinion they should likely be regarded as fortuitous, given that these models did not result in (1) properly spilling waves or (2) correct turbulence/undertow structure at many positions leading up to the inner surf i.e. a correct qualitative description of the breaking process is lacking with these models. (We again emphasize that the undertow in the inner surf zone is primarily dependent on the value of the Wilcox (2006)-type limiter $\lambda_{1}$, and has little to do with the new stabilizing $\lambda_{2}$-limiter introduced in (2.35).)

Upon further inspection of the present results, the quantitative differences in the undertow in the inner surf zone achieved with $\lambda_{1}=0$ (Cases 1 and 3 , dashed lines) and the other models are believed to be related to the waves computed with $\lambda_{1}>0$ (Cases 2, 4 and 5) apparently travelling too fast once the inner surf zone is reached (see 

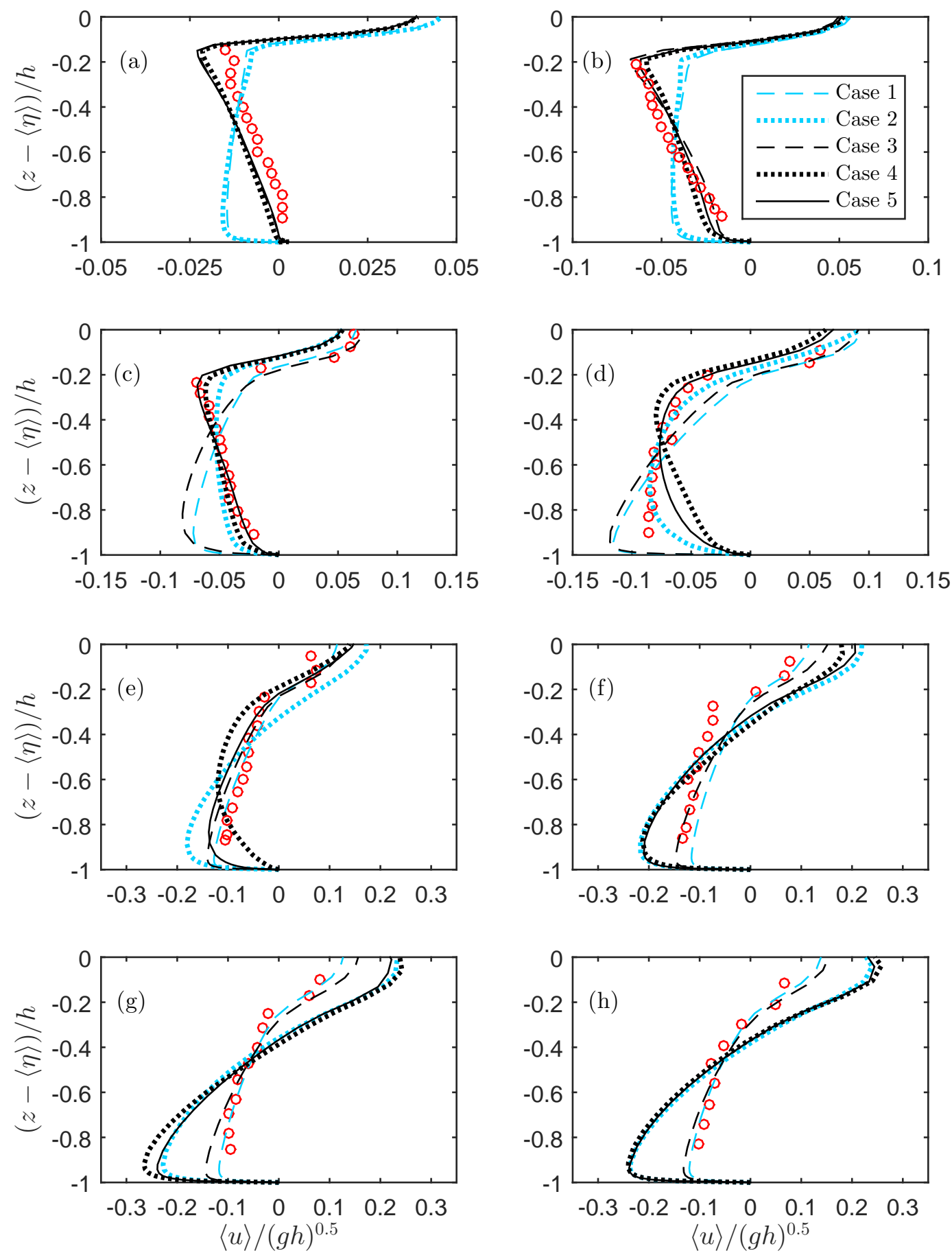

Figure 13. Comparison of modelled (lines) and measured (circles) undertow velocity profiles at (a) $x=-1.265 \mathrm{~m}\left(\tilde{x}=\left(x-x_{b}\right) / h_{b}=-38.518\right)$, (b) $x=5.945 \mathrm{~m}(\tilde{x}=-2.286)$, (c) $x=6.665$ $\mathrm{m}(\tilde{x}=1.332),(\mathrm{d}) x=7.275 \mathrm{~m}(\tilde{x}=4.397),(\mathrm{e}) x=7.885 \mathrm{~m}(\tilde{x}=7.462),(\mathrm{f}) x=8.495 \mathrm{~m}$ $(\tilde{x}=10.528),(\mathrm{g}) x=9.11 \mathrm{~m}(\tilde{x}=13.618)$ and $(\mathrm{h}) x=9.725 \mathrm{~m}(\tilde{x}=16.709)$. 

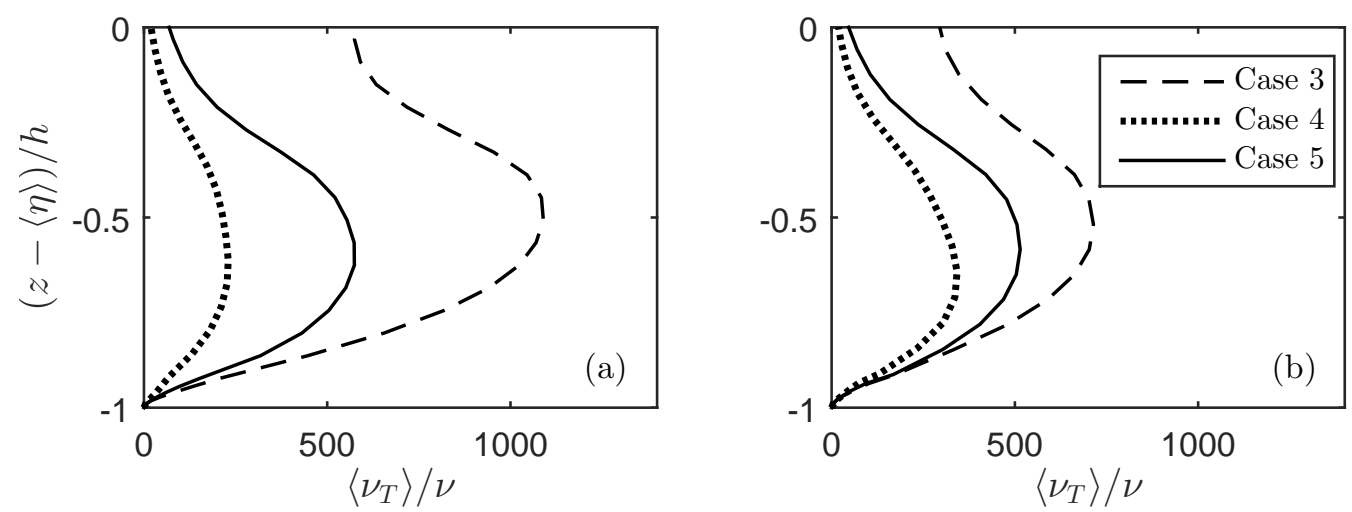

Figure 14. Comparison of modelled eddy viscosity profiles at (a) $x=9.11 \mathrm{~m}$ $\left(\tilde{x}=\left(x-x_{b}\right) / h_{b}=13.618\right)$ and (b) $x=9.725 \mathrm{~m}(\tilde{x}=16.709)$.

again figure 10(c)). A surface roller travelling too fast, as is apparently the case with $\lambda_{1}>0$ as exemplified with Case 5 in figure 10(c), will result in increased flow near the surface. This must be compensated by an increased undertow, hence promoting the exaggerations seen in figure 13(f)-(h) with $\lambda_{1}>0$.

The reason for the slower surface roller in Cases 1 and 3 (sharing $\lambda_{1}=0$ ) relative to the other models is believed to be related to their increased eddy viscosity (hence flow resistance) in the upper part of the flow once in the inner surf zone. Such differences in the eddy viscosity are demonstrated in figure 14, where the average eddy viscosity profiles for Cases 3-5 at the two inner-most positions of the surf zone are shown. The higher eddy viscosity in Case 3 in the upper part of the flow extracts energy from the mean flow, slowing the breaking wave down compared to Case 5, as again shown in figure 10. Based on the locally improved undertow prediction, it seems that Case 3 apparently yields the "correct" eddy viscosity in the inner surf zone. However, this model also clearly over-estimates $k$ in the upper part of the flow, as shown in figure 12(g),(h) (seemingly a remnant of even more severe over-predictions further offshore). In the inner surf zone $\nu_{T} \approx k / \omega$. The implication must then logically be that in Case 3 (similarly, Case 1 ) both $k$ and $\omega$ are locally erroneous, but by approximately the same factor, hence combining to produce an eddy viscosity that is more or less correct. In contrast, the models with $\lambda_{1}>0$ (Cases 2, 4 and 5) yield quite reasonable $k$-profiles in the inner surf zone (figure $12(\mathrm{~g}),(\mathrm{h}))$ implying that only $\omega$ is locally erroneous. How to obtain an accurate eddy viscosity in the upper part of flow in the inner surf zone, while still maintaining a proper description of the breaking process leading up to this region, remains an open question meriting further research.

In summary, of utmost importance, the present case definitively demonstrates that the new stabilized closure proposed herein avoids entirely the important problem of non-physical over-production of turbulence prior to breaking, a long-standing problem in the CFD simulation of surface waves and the primary aim of the present paper. This, in turn, significantly improves both the prediction of turbulent kinetic energy and the evolution of the undertow as waves progress from the shoaling region to the outer surf zone. Conversely, due to their inherent instability, surface waves propagated with existing standard closure models can arrive at the surf zone already polluted, adversely affecting their results locally and extending into the outer surf zone. As intended, results with otherwise identical non-stabilized and stabilized closures become similar once the inner surf zone is reached. Of the five model variations tested, no single model has 
resulted in the best performance in all measured quantities at all positions, with the undertow in the inner surf zone proving the most elusive for the models which seem to give the best description leading up to this region. Nevertheless, having CFD models which begin the breaking process unpolluted by un-physical background turbulence is obviously important in studying the breaking process as a whole. Given that the instability addressed in the present work is inherent within the nearly-potential flow region beneath waves, it is thus recommended that formally stabilized closure models, such as those presented herein, be utilized in future studies involving the RANS-based CFD study of surface waves.

\section{Conclusions}

In this work the instability of two-equation turbulence closure models in a nearlypotential flow region beneath surface waves has been re-visited, a long-standing problem originally diagnosed by Mayer \& Madsen (2000). It has been shown analytically that this problem is widespread and seemingly plagues most (all that the authors have analyzed) commonly used two-equation closure models. These have been demonstrated to be unconditionally (rather than conditionally, as shown by Mayer \& Madsen 2000) unstable, and the asymptotic exponential growth rates for the turbulent kinetic energy and eddy viscosity for several closures have been derived in closed form. Working within the confines of an established stress limiting feature in the $k-\omega$ model, a new and formally stable closure model is proposed. The new closure model, by design, defaults to a desired un-modified $k-\omega$ model in uniform boundary layer flows and other sheared regions, remains true to theoretically-based terms in the $k$-equation, is fully consistent with the Boussinesq approximation, and does not require modification of any standard closure coefficients.

The new stabilized turbulence model has been implemented as closure to a computational fluid dynamics model solving Reynolds-averaged Navier-Stokes (RANS) equations, and directly tested for problems involving both non-breaking and breaking surface waves. As a first idealized test a simple periodic progressive wave train has been considered, which has been kept as close to potential flow as possible. Consistent with analytic expectations, it is demonstrated that the standard $k-\omega$ model results in exponential growth of the turbulent kinetic energy and eddy viscosity, ultimately destroying the simulation by leading to non-physical decay of the wave. Conversely, the new stabilized closure yields an eddy viscosity that decays to insignificant levels, enabling a nearlyconstant form wave propagation over long durations. This test has demonstrated how standard turbulence closure models in wide use can, due to their inherent instability, fail quite spectacularly when applied to even in the simplest of computational surface wave problems. The new stabilized closure, on the other hand, does not adversely affect such simulations.

As a subsequent computational test, the spilling breaking experiment of Ting \& Kirby (1994) has been considered, corresponding to the precise incoming wave conditions where the over-production of turbulence beneath surface waves has been most pronounced in the literature. Consistent with several previous studies, it has been shown that standard closure models can lead to severely over-predicted turbulence levels even prior to wave breaking, with pre-breaking turbulent kinetic energy being the same order of magnitude as within the surf zone. This is not physical, but again a direct consequence of their instability, and implies that standard model results in such applications may well be polluted before the phenomenon of physical interest (i.e. the breaking process) has even begun. It is demonstrated that such pollution results in erroneous structure of 
the undertow velocity profile, both pre-breaking and extending into the outer surf zone. In contrast, the new stabilized closure has been demonstrated to avoid un-physical overproduction of pre-breaking turbulence altogether, and results in a model that is able to produce the correct evolution of the undertow structure from outside to within the surf zone. The new closure model has been demonstrated to predict accurate surface elevation envelopes throughout the breaking process, as well as reasonable turbulence and undertow profiles, especially prior to breaking and in the outer surf zone. The effect of the formal stabilization in the potential flow regions becomes expectedly less important in the inner surf zone, where results of otherwise identical stabilized or non-stabilized models become similar.

While the main text has focused on variations of the $k-\omega$ turbulence closure model, analysis of several other widely used closures (both $k-\omega$ and $k$ - $\varepsilon$ types) are similarly considered in Appendix A. These are likewise demonstrated to be unconditionally unstable, but can fortunately be formally stabilized through similar simple modifications to their stress limiting features. Given the potentially adverse effects of doing otherwise, apparent from several previous studies as well as demonstrated herein, it is recommended that these (or otherwise formally stabilized approaches) be utilized in any future CFD studies involving surface waves based on RANS equations coupled with two-equation closure models. The authors hope that the present study will both raise awareness of this important problem, and that the remedies proposed will enable more accurate simulations of surface waves with such computational models going forward.

\section{Acknowledgements}

The authors acknowledge financial support from the European Union project ASTARTE-Assessment, Strategy And Risk Reduction for Tsunamis in Europe, Grant no. 603839 (FP7-ENV-2013.6.4-3). The authors likewise acknowledge financial support from the Independent Research Fund Denmark project SWASH: Simulating WAve Surf-zone Hydrodynamics and sea bed morphology, Grant no. 8022-00137B. Finally, the authors also thank Prof. Erik Damgaard Christensen for providing valuable feedback on an earlier version of the manuscript.

\section{REFERENCES}

van der A, D. A., van der Zanden, J., O’Donoghue, T., Hurther, D., Caceres, I., McLelland, S. J. \& Ribberink, J. S. 2017 Large-scale laboratory study of breaking wave hydrodynamics over a fixed bar. J. Geophys. Res. 122 (4), 3287-3310.

Baykal, C., Sumer, B. M., Fuhrman, D. R., Jacobsen, N. G. \& Fredsøe, J. 2015 Numerical investigation of flow and scour around a vertical circular cylinder. Phil. Trans. Roy. Soc. A 373, article no. 20140104.

Bayraktar, D., Ahmad, J., Larsen, B. E., Carstensen, S. \& Fuhrman, D. R. 2016 Experimental and numerical study of wave-induced backfilling beneath submarine pipelines. Coast. Eng. 118, 63-75.

Belden, J. \& Techet, A. H. 2011 Simultaneous quantitative flow measurement using PIV on both sides of the air-water interface for breaking waves. Exp. Fluids 50 (1), 149-161.

Berberovic, E., van Hinsberg, N. P., Jakirlic, S., Roisman, I. V. \& Tropea, C. 2009 Drop impact onto a liquid layer of finite thickness: Dynamics of the cavity evolution. Phys. Rev. E 79 (3), article no. 036306.

Bradford, S. F. 2000 Numerical simulation of surf zone dynamics. J. Waterw. Port C-ASCE 126 (1), 1-13.

Bradford, S. F. 2011 Nonhydrostatic model for surf zone simulation. J. Waterw. Port C-ASCE 137 (4), 163-174. 
Brown, S. A., Greaves, D. M., Magar, V. \& Conley, D. C. 2016 Evaluation of turbulence closure models under spilling and plunging breakers in the surf zone. Coast. Eng. 114, 177-193.

Burchard, H. 2002 Applied Turbulence Modelling for Marine Waters. Springer.

Cebeci, T. \& Chang, K. C. 1978 Calculation of incompressible rough-wall boundary-layer flows. AIAA J. 16, 730-735.

Chang, K. A. \& Liu, P. L. F. 1998 Velocity, acceleration and vorticity under a breaking wave. Phys. Fluids 10 (1), 327-329.

Chen, L. F., Zang, J., Hillis, A. J., Morgan, G. C. J. \& Plummer, A. R. 2014 Numerical investigation of wave-structure interaction using OpenFOAM. Ocean Eng. 88, 91-109.

Christensen, E. D. 2006 Large eddy simulation of spilling and plunging breakers. Coast. Eng. $53(5-6), 463-485$.

Deshrande, S. S., Anumolu, L. \& Trujillo, M. F. 2012 Evaluating the performance of the two-phase flow solver interFoam. Comput. Sci. Discov. 5 (1), article no. 014016.

Devolder, B., Rauwoens, P. \& Troch, P. 2017 Application of a buoyancy-modified $k-\omega$ SST turbulence model to simulate wave run-up around a monopile subjected to regular waves using OpenFOAM (R). Coast. Eng. 125, 81-94.

Duncan, J. H., QiaO, H. B., Philomin, V. \& Wenz, A. 1999 Gentle spilling breakers: crest profile evolution. J. Fluid Mech. 379, 191-222.

Durbin, P. A. 2009 Limiters and wall treatments in applied turbulence modeling. Fluid Dyn. Res. 41 (1), article no. 012203.

Fenton, J. D. 1988 The numerical solution of steady water wave problems. Comput. Geosci. 14, 357-368.

Fuhrman, D. R., Baykal, C., Sumer, B. M., Jacobsen, N. G. \& Fredsoe, J. 2014 Numerical simulation of wave-induced scour and backfilling processes beneath submarine pipelines. Coast. Eng. 94, 10-22.

Fuhrman, D. R., Schløer, S. \& Sterner, J. 2013 RANS-based simulation of turbulent wave boundary layer and sheet-flow sediment transport processes. Coast. Eng. 73, 151-166.

Grue, J. \& Jensen, A. 2006 Experimental velocities and accelerations in very steep wave events in deep water. Europ. J. Mech. B/Fluids 25 (5), 554-564.

HaRlow, F. H. \& Welch, J. E. 1965 Numerical calculation of time-dependent viscous incompressible flow of fluid with free surface. Phys. Fluids 8 (12), 2182-2189.

Hieu, P. D., Katsutohi, T. \& CA, V. T. 2004 Numerical simulation of breaking waves using a two-phase flow model. Appl. Math. Mod. 28 (11), 983-1005.

Higuera, P., Lara, J. L. \& Losada, I. J. 2013 Simulating coastal engineering processes with OpenFOAM (R). Coast. Eng. 71, 119-134.

Hirt, C. W. \& Nichols, B.D. 1981 Volume of fluid VOF method for the dynamics of free boundaries. J. Comput. Phys. 39 (1), 201-225.

Hsu, T., Hsien, C., Tsai, C. \& Ou, S. 2015 Coupling VOD/PLIC and embedding method for simulating wave breaking on a sloping beach. J. Marine Sci. Tech.-Taiwan 23 (4), $498-507$.

Hsu, T. J., Sakakiyama, T. \& Liu, P. L. F. 2002 A numerical model for wave motions and turbulence flows in front of a composite breakwater. Coast. Eng. 46 (1), 25-50.

Hu, Z. Z., Greaves, D. \& Raby, A. 2016 Numerical wave tank study of extreme waves and wave-structure interaction using OpenFoam (R). Ocean Eng. 126, 329-342.

Jacobsen, N. G., Fredsøe, J. \& Jensen, J. H. 2014 Formation and development of a breaker bar under regular waves. Part 1: Model description and hydrodynamics. Coast. Eng. 88, $182-193$.

Jacobsen, N. G., Fuhrman, D. R. \& Fredsøe, J. 2012 A wave generation toolbox for the open-source CFD library: OpenFOAM (R). Int. J. Numer. Meth. Fluids 70, 1073-1088.

Jacobsen, N. G., van Gent, M. R. A. \& Wolters, G. 2015 Numerical analysis of the interaction of irregular waves with two dimensional permeable coastal structures. Coast. Eng. 102, 13-29.

Kimmoun, O. \& Branger, H. 2007 A particle image velocimetry investigation on laboratory surf-zone breaking waves over a sloping beach. J. Fluid Mech. 588, 353-397.

LARsen, B. E. 2018 Tsunami-seabed interactions. PhD thesis, Technical University of Denmark, Kgs. Lyngby, Denmark. 
Larsen, B. E., Arbøll, L. K., Frigaard, S., Carstensen, S. \& Fuhrman, D. R. 2018 a Experimental study of tsunami-induced scour around a monopile foundation. Coast. Eng. 138, 9-21.

Larsen, B. E., Fuhrman, D. R., Baykal, C. \& Sumer, B. M. 2017 Tsunami-induced scour around monopile foundations. Coast. Eng. 129, 36-49.

Larsen, B. E., Fuhrman, D. R. \& Roenby, J. $2018 b$ Performance of interFoam on the simulation of progressive waves. axiv.org. ID: 1804.01158 .

Larsen, B. E., Fuhrman, D. R. \& Sumer, B. M. 2016 Simulation of wave-plus-current scour beneath submarine pipelines. J. Waterw. Port C-ASCE 142 (5), article no. 08216001.

Launder, B. E. \& Sharma, B. I. 1974 Application of the energy-dissipation model or turbulence to the calculation of flow near a spinning disc. Lett. Heat Mass Transfer 1 (2), 131-7, 131-137.

Lemos, C. M. 1992 Wave breaking: A numerical study. In Lecture Notes in Engineering, , vol. 71, pp. 1-185. Berlin: Springer.

Lin, P. Z. \& LiU, P. L. F. 1998 A numerical study of breaking waves in the surf zone. J. Fluid Mech. 359, 239-264.

Makris, C. V., Memos, C. D. \& Krestenitis, Y. N. 2016 Numerical modeling of surf zone dynamics under weakly plunging breakers with sph method. Ocean Model. 98, 12-35.

Mayer, S. \& MAdSen, P. A. 2000 Simulations of breaking waves in the surf zone using a Navier-Stokes solver. In Proc. 25th Int. Conf. Coast. Eng., pp. 928-941. Sydney, Australia.

Menter, F. R. 1994 Two-equation eddy-viscosity turbulence models for engineering applications. AIAA J. 32 (8), 1598-1605.

Paulsen, B. T., Bredmose, H., Bingham, H. B. \& Jacobsen, N. G. 2014 Forcing of a bottom-mounted circular cylinder by steep regular water waves at finite depth. J. Fluid Mech. 755, 1-34.

RodI, W. 1987 Examples of calculation methods for flow and mixing in stratified fluids. J. Geophys. Res. 92 (C5), 5305-5328.

Roenby, J., Larsen, B. E., Bredmose, H. \& Jasak, H. 2017 A new volume-of-fluid method in OpenFOAM. In 7th Int. Conf. Comput. Methods Marine Eng., pp. 1-12. 7th Int. Conf. Comput. Methods Marine Eng. Nantes, France.

Ruessink, B. G., van den Berg, T. J. J. \& van Rijn, L. C. 2009 Modeling sediment transport beneath skewed asymmetric waves above a plane bed. J. Geophys. Res. 114, article no. C11021.

Sakai, T., Mizutani, T. TAnaka, H. \& Tada, Y. 1986 Vortex formation in plunging breakers. In Proc. 20th Conf. Coast. Eng., pp. 711-723. Taipei, Taiwan.

Schmitt, P. \& Elsaesser, B. 2015 On the use of OpenFOAM to model oscillating wave surge converters. Ocean Eng. 108, 98-104.

Schumann, U. \& Gerz, T. 1995 Turbulent mixing in stably stratified shear flows. J. Appl. Meteorol. 34 (1), 33-48.

Scott, C. P., Cox, D. T., Maddux, T. B. \& Long, J. W. 2005 Large-scale laboratory observations of turbulence on a fixed barred beach. Meas. Sci. Technol. 16 (10), 19031912.

ShaO, S. 2006 Simulation of breaking wave by SPH method coupled with k- $\epsilon$ model. J. Hydraul. Res. 44 (3), 338-349.

Stive, M. J. F. \& Wind, H. G. 1982 A study of radiation stress and set-up in the nearshore region. Coast. Eng. 6 (1), 1-25.

Svendsen, I. A. 1987 Analysis of surf zone turbulence. J. Geophys. Res. 92 (C5), 5115-5124.

Ting, F. C. K. \& Kirby, J. T. 1994 Observation of undertow and turbulence in a laboratory surf zone. Coast. Eng. 24 (1-2), 51-80.

Ting, F. C. K. \& Kirby, J. T. 1996 Dynamics of surf-zone turbulence in a spilling breaker. Coast. Eng. 27 (3-4), 131-160.

Townsend, A.A. 1976 The Structure of Turbulent Shear Flow. Cambridge University Press.

Umlauf, L., Burchard, H. \& Hutter, K. 2003 Extending the $k$ - $\omega$ turbulence model towards oceanic applications. Ocean Model. 5 (3), 195-218.

Watanabe, Y. \& SAeki, H. 1999 Three-dimensional large eddy simulation of breaking waves. Coast. Eng. J. 41 (3-4), 281-301. 
Wilcox, D. C. 1988 Reassessment of the scale-determining equation for advanced turbulence models. AIAA J. 26 (11), 1299-1310.

Wilcox, D. C. 2006 Turbulence Modeling for CFD, 3rd edn. La Canada, California: DCW Industries, Inc.

Wilcox, D. C. 2008 Formulation of the $k$ - $\omega$ turbulence model revisited. AIAA J. 46, 2823-2838.

XIE, Z. 2013 Two-phase flow modelling of spilling and plunging breaking waves. Appl. Math. Model. 37 (6), 3698-3713.

Yakhot, V., Thangam, S., Speziale, C. G., Orszag, S. A. \& Gatski, T. B. 1991 Development of turbulence models for shear flows by a double expansion technique. Inst. Computer Application Sci. Eng. NAS1-18605.

\section{Appendix A. Analysis of additional turbulence closure models}

In this appendix three additional popular two-equation turbulence closure models are analyzed for stability in a region of nearly-potential flow with finite strain. It is demonstrated that, in their standard forms, these models are likewise unconditionally unstable. Moreover, it is shown that each may be formally stabilized via the addition of similar stress-limiting modifications, as devised in $\S 2.6$. It is recommended that the stabilized versions of these these models be utilized in any future CFD simulations of free surface waves, to avoid non-physical exponential growth of the turbulent kinetic energy and eddy viscosity in the nearly-potential flow region, the significant benefits of which have been demonstrated in the main text.

\section{A.1. $k-\omega$ SST model}

In addition to the standard $k-\omega$ models considered in the main text, another widely used variant is the $k$ - $\omega$ SST (shear stress transport) model of Menter (1994). Neglecting convective and diffusive terms as before, in this model the $k$ equation is

$$
\frac{\partial k}{\partial t}=\min \left(\nu_{T} p_{0}, c_{1} \beta^{*} \omega k\right)-\beta^{*} \omega k,
$$

combined with the $\omega$ equation from (2.18), from which it is evident that $\omega$ will ultimately tend to $\omega_{\infty}$ from $(2.20)$. In this model the eddy viscosity is defined by

$$
\nu_{T}=\frac{a_{1} k}{\max \left(a_{1} \omega, F_{2} \sqrt{p_{0}}\right)}
$$

where the denominator includes a stress limiting feature somewhat similar to that used in the Wilcox (2006) model. In the above $c_{1}=10, a_{1}=0.31$, and $\alpha$ and $\beta$ are closure coefficients, which are a blend of inner (subscript 1) and outer constants (subscript 2) based on

$$
\phi=F_{1} \phi_{1}+\left(1-F_{1}\right) \phi_{2},
$$

where $\phi$ represents either of these coefficients. The inner coefficients are $\alpha_{1}=0.5532$ and $\beta_{1}=0.075$, whereas the outer coefficients correspond to $\alpha_{2}=0.4403 \beta_{2}=0.0828$. This model utilizes the following two blending functions:

$$
\begin{aligned}
& \Lambda_{1}=\min \left\{\max \left(\frac{\sqrt{k}}{\beta^{*} \omega z_{w}}, \frac{500 \nu}{z_{w}^{2} \omega}\right), 10\right\}, \\
& \Lambda_{2}=\min \left\{\max \left[\frac{2 \sqrt{k}}{\beta^{*} \omega z_{w}}, \frac{500 \nu}{z_{w}^{2} \omega}\right], 100\right\},
\end{aligned}
$$


where $F_{1}=\tanh \left(\Lambda_{1}^{4}\right)$ and $F_{2}=\tanh \left(\Lambda_{2}^{2}\right)$, and $z_{w}$ represents the distance to the nearest wall.

Due to the blending of inner and outer constants, the min condition in the production term in the $k$ equation (A 1) and the max condition in $\nu_{T}$ (A 2), the analysis of the $k-\omega$ SST model is not as simple as in the standard $k$ - $\omega$ model variations. It is therefore necessary to split the analysis into three different cases: First, if the first arguments in (A 1) and (A 2) are active, then this model is the same as the standard $k-\omega$ model, and hence will result in exponential growth at the rate predicted analytically by (2.22). As this rate is inevitably positive (i.e. regardless if inner or outer coefficients are used) the model will be unstable, and it is hence clear that the model will eventually tend to the inner coefficients, yielding $F_{1}=1$ and $\Gamma_{\infty} \approx 0.124 \sqrt{p_{0}}$. Second, suppose that the second term in the max condition of (A 2) is active. Inserting the threshold $\omega=\omega_{\infty}$ into the $k$ equation (A 1) yields

$$
\frac{1}{k} \frac{\partial k}{\partial t}=\Gamma_{\infty}, \quad \Gamma_{\infty}=\sqrt{p_{0}}\left(\frac{a_{1}}{F_{2}}-\frac{\sqrt{\alpha} \beta^{*}}{\sqrt{\beta}}\right) .
$$

Here $\Gamma_{\infty}$ is again inevitably positive, and thus inserting $F_{2}=1$ and the inner coefficient values yields $\Gamma_{\infty} \approx 0.0656 \sqrt{p_{0}}$. Third, suppose that the second term in the min condition (A 1) is active. Inserting $\omega=\omega_{\infty}$ into (A 1) then similarly yields the growth rate

$$
\Gamma_{\infty}=\left(c_{1}-1\right) \beta^{*} \sqrt{\frac{\alpha}{\beta}} \sqrt{p_{0}} .
$$

This is also inevitably positive, and inserting inner coefficients yields $\Gamma_{\infty} \approx 2.20 \sqrt{p_{0}}$.

To conclude, the $k$ - $\omega$ SST model is also unconditionally unstable, with an asymptotic growth rate that is at least $\Gamma_{\infty} \approx 0.0656 \sqrt{p_{0}}$, though it may exhibit preliminary exponential growth that is considerably larger. To independently demonstrate the validity of the analysis above, the two differential equations (A 1) and (2.18) (complete with the blending functions above, utilizing $z_{w}=10 \sqrt{k_{0} p_{0}}$ ) are again solved numerically, with the same initial conditions as in $\S 2.3$ (these are maintained throughout the Appendix). The result for $\nu_{T} / \nu$ of the numerical solution is shown in figure 15(a), where it is seen that after the initial evolution, the model (full line) is unstable at an accelerated rate, before ultimately arriving at the asymptotic $\Gamma_{\infty} \approx 0.0656 \sqrt{p_{0}}$ (dashed line) predicted above.

Similar to the standard variants, the $k-\omega$ SST model can be stabilized via a slight modification to the stress limiting feature. In this case the necessary modification corresponds e.g. to re-defining the eddy viscosity from (A 2) according to

$$
\nu_{T}=\frac{a_{1} k}{\max \left(a_{1} \omega, F_{2} \sqrt{p_{0}}, a_{1} \lambda_{2} \frac{\beta}{\beta^{*} \alpha} \frac{p_{0}}{p_{\Omega}} \omega\right)}
$$

where a new (third) argument has been added within the max function, designed to only be active in a region of nearly-potential flow i.e. where $p_{\Omega} \ll p_{0}$. Adopting this value within $\nu_{T}$, and repeating the analysis above leads to the asymptotic growth rate

$$
\Gamma_{\infty}=\frac{\left(p_{\Omega}-\lambda_{2} p_{0}\right) \beta^{*}}{\lambda_{2}} \sqrt{\frac{\alpha}{p_{0} \beta}} .
$$

This is formally stable provided that $p_{\Omega} / p_{0} \leqslant \lambda_{2}$, in accordance with $(2.38)$, such that $\lambda_{2}$ defines the effective potential flow threshold, as before. Note that at the pure potential flow limit (where $p_{\Omega}=0$ ) the growth (or in this case, decay) rate is $\Gamma_{\infty} \approx-0.244 \sqrt{p_{0}}$. Exponential decay in line with that predicted by (A 9) is independently confirmed by numerical solution of the reduced modified model equations in figure 16(a). 

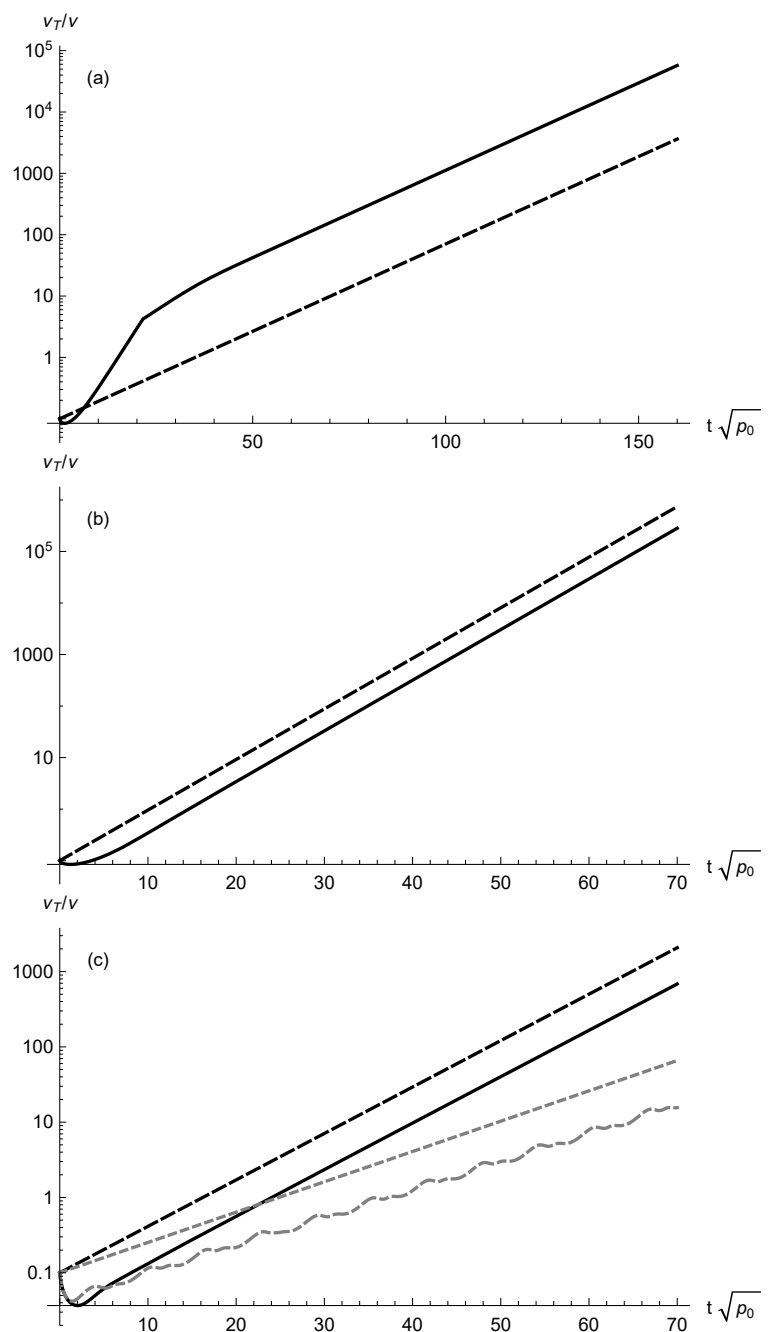

Figure 15. Simulated development of $\nu_{T} / \nu$ (full lines) compared to the asymptotic exponential growth $\exp \left(\Gamma_{\infty} t\right)$ (dashed lines) for the (a) $k-\omega \mathrm{SST}$, (b) $k-\varepsilon$, and (c) RNG $k-\varepsilon$ models. In (c) the grey dashed-dotted line presents numerical results when an oscillation in the production is added as $p_{0}+\tilde{p}_{0} \sin \left(\sqrt{p_{0}} t\right)$ and the grey dotted line depicts the predicted exponential growth with $\Gamma_{\infty}$ calculated analytically from (A 21) with $\omega=\omega_{\infty}$ from (A 22). After some initial development all numerical results are seen to follow precisely the analytically predicted asymptotic exponential growth rates.

\section{A.2. $k-\varepsilon$ model}

Another widely used class of turbulence closure models are those in the $k-\varepsilon$ family. Neglecting convective and diffusive terms, the standard high Reynolds number $k-\varepsilon$ model of Launder \& Sharma (1974) reduces to the following two equations

$$
\begin{gathered}
\frac{\partial k}{\partial t}=\nu_{T} p_{0}-\varepsilon, \\
\frac{\partial \varepsilon}{\partial t}=C_{1} C_{\mu} k p_{0}-\frac{C_{2} \varepsilon^{2}}{k},
\end{gathered}
$$



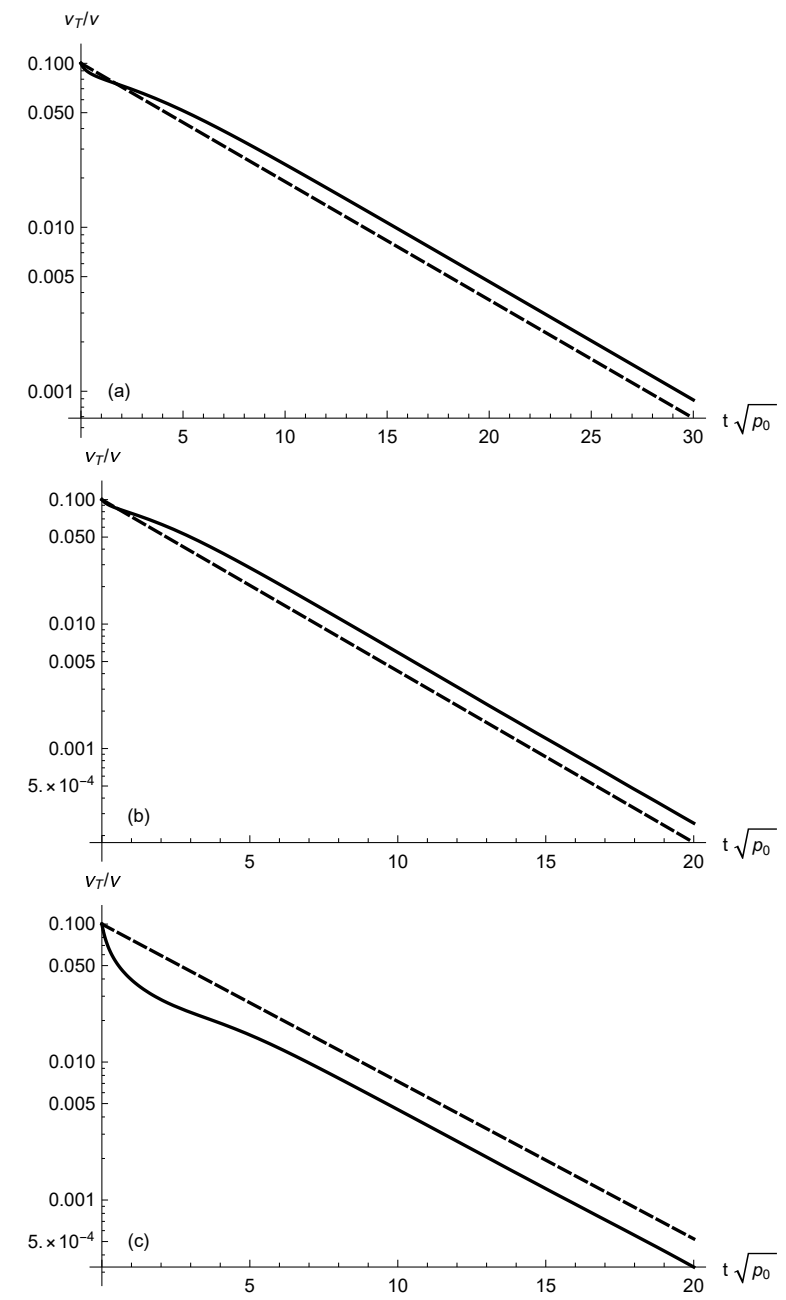

FIGURE 16. Simulated development of $\nu_{T} / \nu$ (full lines) compared to the predicted exponential decay $\exp \left(\Gamma_{\infty} t\right)$ (dashed lines) for stabilized versions of the (a) $k-\omega$ SST, (b) $k-\varepsilon$, and (c) RNG $k-\varepsilon$ models. After some initial development all numerical results are seen to follow precisely the analytically predicted asymptotic exponential decay rates.

where the eddy viscosity is defined as

$$
\nu_{T}=\frac{C_{\mu} k^{2}}{\varepsilon} .
$$

It is emphasized that, similar to the $k-\omega$ models considered previously, the eddy viscosity is here retained as a variable only within the $k$ equation; It has been explicitly eliminated within the $\varepsilon$ equation by invoking the definition (A 12). This makes no difference to the model in its standard form, but is an important detail in its formal stabilization, to be presented in what follows. The closure coefficients are $C_{\mu}=0.09, C_{1}=1.44$ and $C_{2}=1.92$.

To analyze the stability of this model, it turns out to be convenient to utilize an equivalent equation for the specific dissipation rate $\omega=\varepsilon /\left(C_{\mu} k\right)$ :

$$
\frac{\partial \omega}{\partial t}=\frac{1}{C_{\mu} k} \frac{\partial \varepsilon}{\partial t}-\frac{\varepsilon}{C_{\mu} k^{2}} \frac{\partial k}{\partial t}=\left(C_{1}-1\right) p_{0}-C_{\mu}\left(C_{2}-1\right) \omega^{2} .
$$


Although it is not directly modelled, this variable will still (regardless of the initial conditions) evolve asymptotically to the constant

$$
\omega_{\infty}=\sqrt{\frac{\left(C_{1}-1\right) p_{0}}{C_{\mu}\left(C_{2}-1\right)}} \approx 2.31 \sqrt{p_{0}}
$$

such that $\partial \omega / \partial t=0$. Substituting $\varepsilon=C_{\mu} \omega k$ with $\omega=\omega_{\infty}$ back into (A 10) and (A 11) leads to linearized equations of the form (2.21) where the unstable growth rate is

$$
\Gamma_{\infty}=\frac{C_{\mu}\left(C_{2}-C_{1}\right) \sqrt{p_{0}}}{\sqrt{\left(C_{1}-1\right)\left(C_{\mu}\left(C_{2}-1\right)\right)}} \approx 0.226 \sqrt{p_{0}} .
$$

Hence, similar to the pre-existing $k-\omega$ models considered previously, the standard $k-\varepsilon$ model is likewise unconditionally unstable for the conditions considered. The analysis above is confirmed through independent numerical simulation of (A 10) and (A 11), the results of which are shown in figure $15(\mathrm{~b})$.

It can be noted that Mayer \& Madsen (2000) stated, but did not directly show, that the standard $k-\varepsilon$ model would also have conditional uncontrollable growth of $\nu_{T}$ when used to simulate surface waves. The analysis above demonstrates this formally, and extends their conditional finding to be unconditional. The instability of the $k$ - $\varepsilon$ model is also widely evidenced in simulation of surface waves, with the model resulting in severely overpredicted turbulent kinetic energy, especially pre-breaking as shown by several authors e.g. Bradford (2000) and Xie (2013)

The standard $k-\varepsilon$ model presented above can be formally stabilized via the following simple modification to the eddy viscosity:

$$
\nu_{T}=\frac{C_{\mu} k^{2}}{\tilde{\varepsilon}}, \quad \tilde{\varepsilon}=\max \left(\varepsilon, \lambda_{2} \frac{C_{2}}{C_{1}} \frac{p_{0}}{p_{\Omega}} \varepsilon\right)
$$

In most circumstances (sheared flow regions) the first argument in the max function will be active, and the model is then identical to the standard $k-\varepsilon$ model. In a region of nearly-potential flow, however, the second argument is designed to become active. Letting $\tilde{\varepsilon}$ take this value, and repeating the analysis above leads to the growth rate

$$
\Gamma_{\infty}=\frac{\left(p_{\Omega}-\lambda_{2} p_{0}\right) C_{\mu}}{\sqrt{\lambda_{2} C_{2} p_{0}-p_{\Omega}}} \sqrt{\frac{C_{1} C_{2}}{\lambda_{2}\left(C_{\mu}\left(C_{2}-1\right)\right)}} .
$$

This is formally stable provided that $p_{\Omega} / p_{0} \leqslant \lambda_{2}$, where $\lambda_{2}$ again defines the effective potential flow threshold. Note that the singularity in (A 17) at $p_{\Omega} / p_{0}=C_{2} \lambda_{2}=1.92 \lambda_{2}$ is outside the range $p_{\Omega} / p_{0}<C_{2} \lambda_{2} / C_{1} \approx 1.33 \lambda_{2}$, where the new limiter introduced in (A 16) is active. Note also that at the limit where $p_{\Omega}=0$ the decay rate is $\Gamma_{\infty} \approx-0.375 \sqrt{p_{0}}$. Exponential decay of the eddy viscosity in accordance with (A 17) is demonstrated via independent numerical solution of (A 10) and (A 11), while invoking (A 16), in figure 16(b) using the same initial conditions as before.

The strategy described above to stabilize a $k-\varepsilon$ type model is slightly similar to that employed by Hsu et al. (2002), who used a damping function to decrease the eddy viscosity in "low turbulence" regions. The solution suggested above, however, is fundamentally different in that it is based directly on analysis of the problem at hand. 


\section{A.3. $R N G k-\varepsilon$ model}

Neglecting convective and diffusive terms as before, the RNG $k$ - $\varepsilon$ model of Yakhot et al. (1991) is again comprised of (A 10) and (A 11), but with $C_{1}$ now defined as

$$
C_{1}=C_{1 \varepsilon}-\frac{\eta_{r n g}\left(1-\eta_{r n g} / \eta_{0}\right)}{1+\beta_{r n g} \eta_{r n g}^{3}}
$$

where $\eta_{0}=4.38, \beta_{r n g}=0.012$, and $\eta_{r n g}=\sqrt{p_{0}} k / \varepsilon$, with closure coefficients $C_{\mu}=0.0845$, $C_{1 \varepsilon}=1.42$ and $C_{2}=1.68$. The eddy viscosity is again defined according to (A 12 ). Due to the added complexity of this model our analysis will be performed with all coefficient values invoked. Similar to before, we invoke the above into the equivalent $\omega$ equation (A 13), which in this case leads to a complicated polynomial in $\omega$. To find the asymptotic value $\omega=\omega_{\infty}$ we set $\partial \omega / \partial t=0$ and look for solutions of the form

$$
\omega_{\infty}=A \sqrt{p_{0}} .
$$

After some simplification, this ultimately leads to the following fifth-order polynomial:

$$
A^{5}-c_{3} A^{3}+225.846 A^{2}-556.477 A-c_{0}=0
$$

where $c_{3}=7.30943$ and $c_{0}=145.377$. This has the lone physical (real and positive) root $A=2.702$. Invoking this back into (A 10) and (A 11) then leads to linearized equations of the form (2.21), with unstable growth rate

$$
\Gamma_{\infty}=\frac{p_{0}}{\omega_{\infty}}-C_{\mu} \omega_{\infty}
$$

yielding $\Gamma_{\infty} \approx 0.142 \sqrt{p_{0}}$. Hence, this model is also unconditionally unstable. The unstable growth rate found analytically above is confirmed via independent numerical simulation of (A 10) and (A 11), after invoking (A 18), in figure 15(c).

Interestingly, in the study of breaking waves by Brown et al. (2016), the RNG $k-\varepsilon$ model was the only closure model of those tested not to result in excessive turbulence prior to breaking. To investigate the potential reasons for this observation, we have extended the analysis above to also consider the addition of an oscillatory production component i.e. $p_{0}+\tilde{p}_{0} \sin (\sigma t)$. Repeating the analysis above, it can be shown that, to leading-order in $\tilde{p}_{0}$, the addition of the oscillating components modifies the (now period-averaged) asymptotic value for $\omega$ to

$$
\left\langle\omega_{\infty}\right\rangle \approx 2.702 \sqrt{p_{0}+0.1795 \frac{\tilde{p}_{0}^{2}}{p_{0}}},
$$

which is obviously an extension of the steady-state result (A 19). We have likewise simulated such a case numerically, taking $\sigma=\sqrt{p_{0}}=\sqrt{\tilde{p}_{0}}$ for simplicity, and the resulting time evolution of the eddy viscosity is depicted as the dashed-dotted line in figure 15 (c). From (A 22) this case yields $\left\langle\omega_{\infty}\right\rangle \approx 2.93 \sqrt{p_{0}}$, which when inserted back into (A 21) results in the predicted period-averaged growth rate $\left\langle\Gamma_{\infty}\right\rangle \approx 0.0927 \sqrt{p_{0}}$. While still positive, this is considerably less than would be expected from the strictly steadystate analysis from either the standard or RNG $k-\varepsilon$ models. This predicted exponential growth is likewise depicted on figure $15(\mathrm{c})$ as the dotted line, which matches nearly perfectly the long-term (period-averaged) growth rate exhibited by the independent numerical simulation. Hence, this extension of the steady-state analysis (as well as the simple numerical simulation) likely demonstrates why reduced growth rates in the CFD simulation of surface waves have seemingly been observed in practice with the RNG $k-\varepsilon$ model. Note that similarly adding oscillatory components to $p_{0}$ e.g. in the numerical 
simulation of the reduced standard $k-\varepsilon$ or $k-\omega$ models does not lead to significant deviations from the growth rates $\Gamma_{\infty}$ predicted by the steady-state linear stability theory. Hence, this behaviour is seemingly a rather unique feature of the RNG $k-\varepsilon$ model, and can clearly be attributed to the modified term in the $\varepsilon$ equation. Nevertheless, despite the potentially reduced growth, this closure is still formally unconditionally unstable at the steady-state limit, and its performance as a whole for simulating surface waves leaves much to be desired. For example, it was ranked as the least accurate of all of the closure models tested by Brown et al. (2016), severely overestimating turbulence levels in the inner surf zone.

In any event, similar to the standard $k-\varepsilon$ model, the RNG $k-\varepsilon$ model can be formally stabilized via the following simple modification to the eddy viscosity:

$$
\nu_{T}=\frac{C_{\mu} k^{2}}{\tilde{\varepsilon}}, \quad \tilde{\varepsilon}=\max \left(\varepsilon, 1.2603 \lambda_{2} \frac{C_{2}}{C_{1 \varepsilon}} \frac{p_{0}}{p_{\Omega}} \varepsilon\right) .
$$

As before, in most circumstances (sheared flow regions) the first term in the max argument will be active, and the model is then identical to the standard RNG $k-\varepsilon$ model, whereas in a region of nearly-potential flow the second term will be active. Letting $\tilde{\varepsilon}$ take this value, we will seek a solution for the (steady-state) asymptotic value value for $\omega$ of the form

$$
\omega_{\infty} \approx A \sqrt{p_{0}+B \frac{p_{\Omega}}{\lambda_{2}}}
$$

To accomplish this we set $\partial \omega / \partial t=0$, and Taylor expand the resulting $\omega$ equation about $p_{\Omega}=0$. Collecting $O(1)$ terms leads, after some simplification, to a polynomial of the form (A 20), now with $c_{0}=491.512$ and $c_{3}=24.7128$. This has the lone physical (real and positive) root $A=3.716$. Inserting this value for $A$ and then requiring that $O\left(p_{\Omega}\right)$ terms vanish subsequently yields $B=-0.425254$, thus defining the leading-order contributions for $\omega_{\infty}$. Inserting $\omega=\omega_{\infty}$ from (A 24) back into (A 10) and (A 11) leads to linearized equations of the form $(2.21)$, where the asymptotic growth rate is

$$
\Gamma_{\infty}=\frac{0.3140\left(p_{\Omega}-\lambda_{2} p_{0}\right)}{\lambda_{2} \sqrt{p_{0}-\frac{0.4253 p_{\Omega}}{\lambda_{2}}}} .
$$

This is again formally stable provided that $p_{\Omega} / p_{0} \leqslant \lambda_{2}$, where $\lambda_{2}$ again defines the effective potential flow threshold. Note that the singularity in (A 25) at $p_{\Omega} / p_{0} \approx 2.351 \lambda_{2}$ is outside the range $p_{\Omega} / p_{0}<1.2603 C_{2} / C_{1 \varepsilon} \lambda_{2} \approx 1.49 \lambda_{2}$ where the new limiter introduced in (A 23) is active. Note also that at the limit where $p_{\Omega}=0$ the decay rate is $\Gamma_{\infty} \approx-0.314 \sqrt{p_{0}}$. Exponential decay in accordance with (A 25) is demonstrated via independent numerical solution of the modified RNG $k-\varepsilon$ equations, i.e. after invoking (A 23), in figure 16(c).

\section{Appendix B. Derivation of the buoyancy production closure coefficient $\alpha_{b}^{*}$}

To derive a value for the buoyancy production closure coefficient $\alpha_{b}^{*}$, consider a sheared flow region such that $\tilde{\omega}=\tilde{\tilde{\omega}}$. Neglecting convective and diffusive terms, but retaining shear and buoyancy production terms, we may reduce (2.1) and (2.2) to

$$
\frac{\partial k}{\partial t}=p_{0} \nu_{T}-\alpha_{b}^{*} N^{2} \nu_{T}-\beta^{*} k \omega
$$




$$
\frac{\partial \omega}{\partial t}=\alpha \frac{\omega}{k} p_{0} \nu_{T}-\beta \omega^{2}
$$

where the eddy viscosity is intentionally kept general. To find steady state conditions, we set both equations above equal to zero, and solve for $p_{0}$ and $N^{2}$. This leads to the steady-state Richardson number

$$
R i_{\infty}=\frac{N^{2}}{p_{0}}=\frac{\beta-\alpha \beta^{*}}{\alpha_{b}^{*} \beta} \approx \frac{0.339}{\alpha_{b}^{*}} .
$$

According to Schumann \& Gerz (1995) (see also Burchard 2002) the constraint $R i_{\infty} \leqslant$ 0.25 should be satisfied, implying that, at minimum, we must require $\alpha_{b}^{*} \approx 1.36$, which is the value adopted throughout the present work. Note that this is quite similar to the value $1 / 0.7 \approx 1.4$ used by several other authors (e.g. Rodi 1987; Ruessink et al. 2009; Fuhrman et al. 2013), and conveniently enables the constraint above to be satisfied without requiring an additional buoyancy production closure term in the $\omega$ equation. 